\title{
Introduction to the Intersection Theory for Twisted Homology and Cohomology Groups
}

\author{
Keiji Matsumoto* \\ Department of Mathematics, Faculty of Science, Hokkaido University, \\ Kita10-Nishi8, Kita-ku, Sapporo, 060-0810, Japan \\ E-mail: matsu@math.sci.hokudai.ac.jp
}

We give an introduction to the intersection theory for twisted homology and cohomology groups associated with Euler type integrals of hypergeometric functions. We introuduce twisted homology and cohomology groups motivated by Twisted Stokes' Theorem, and give their dimension formulas. We define an intersection form between twisted homology groups and that between twisted cohomology groups, and explain how to compute them. These intersection forms are compatible with the natural pairing between the twisted homology and cohomology groups. This compatibility yields a twisted period relation, which relates intersection numbers and period integrals regarded as some kinds of hypergeometric functions. In Appendix, we show that Elliott's identity can be obtained from the twisted period relation.

MathemAmplitudes 2019: Intersection Theory \& Feynman Integrals (MA2019)

18-20 December 2019

Padova, Italy

* Speaker 


\section{Contents}

1 Introduction 2

2 Regularization of open intervals $\quad 4$

3 Lauricella's hypergeometric system $\mathcal{F}_{D}$

4 Twisted Stokes' Theorem $\quad 7$

5 Twisted homology groups 9

6 Intersection form between twisted homology groups 10

7 Twisted cohomology groups 12

8 Intersection form between twisted cohomology groups 16

$9 \begin{array}{ll}\text { Twisted period relation } & 18\end{array}$

A Elliott's identity as the twisted period relation $\quad 22$

A.1 Integral representations $\quad 22$

A.2 Setting of a local system 23

A.3 Transform of a twisted period relation into Elliott's identity 24

$\begin{array}{lr}\text { Acknowledgments } & 25\end{array}$

\section{Introduction}

This article is an introduction to the intersection theory for twisted homology and cohomology groups based on results in [AK], [CM], [KY1], [M1] and [Y2].

The hypergeometric series $F(a, b, c ; x)$ is defined by

$$
F(a, b, c ; x)=\sum_{n=0}^{\infty} \frac{(a)_{n}(b)_{n}}{(c)_{n} n !} x^{n},
$$

where $x$ is a main variable in $\mathbb{D}=\{x \in \mathbb{C}|| x \mid<1\}, a, b, c$ are complex parameters with $c \neq 0,-1,-2, \ldots$, and

$$
(a)_{n}=a(a+1) \cdots(a+n-1), \quad(a)_{0}=1 .
$$

Under $\operatorname{Re}(a), \operatorname{Re}(c-a)>0, F(a, b, c ; x)$ admits an Euler type integral

$$
F(a, b, c ; x)=\frac{\Gamma(c)}{\Gamma(a) \Gamma(c-a)} \int_{0}^{1} t^{a}(1-t)^{c-a}(1-t x)^{-b} \frac{d t}{t(1-t)} .
$$


Here we assign a branch of the multi-valued function $u(t)=t^{a}(1-t)^{c-a}(1-t x)^{-b}$ in (1) on the integration interval $(0,1)$ by $\arg (t)=\arg (1-t)=0$, and $-\pi / 2<\arg (1-t x)<\pi / 2$ for $x \in \mathbb{D}$. We separate $u(t)=t^{a}(1-t)^{c-a}(1-t x)^{-b}$ and $\varphi=\frac{d t}{t(1-t)}$ from the integrand in (1), and regard this integral as a pairing between the 1 -form $\varphi$ and a pair $I \otimes u_{I}(t)$ of $I=(0,1)$ and a branch of $u_{I}(t)=t^{a}(1-t)^{c-a}(1-t x)^{-b}$ on $I$. With respect to this, any branch $u_{I}(t)$ on $I$ vanishes at the boundary of $I$ under $\operatorname{Re}(a), \operatorname{Re}(c-a)>0$, and the exterior derivative $d$ acts on $u(t) \varphi$ as

$$
d(u(t) \varphi)=u(t) \cdot d \varphi+d u(t) \wedge \varphi=u(t) \cdot\left(d \varphi+\frac{d u(t)}{u(t)} \wedge \varphi\right)=0 .
$$

Motivated by the above, we define twisted homology and cohomology groups associated with an Euler type integral of Lauricella's hypergeometric series $F_{D}$ in $m$-variables given in (8) as follows. We set a multi-valued function

$$
u(t)=t^{\alpha_{0}}\left(t-x_{1}\right)^{\alpha_{1}} \cdots\left(t-x_{m}\right)^{\alpha_{m}}(t-1)^{\alpha_{m+1}}
$$

on the space $T_{x}=\mathbb{P}^{1}-\left\{x_{0}, x_{1}, \ldots, x_{m}, x_{m+1}, x_{m+2}\right\}$ for mutually different fixed complex variables $x_{0}=0, x_{1}, \ldots, x_{m}, x_{m+1}=1, x_{m+2}=\infty$ with parameters

$$
\alpha=\left(\alpha_{0}, \alpha_{1}, \ldots, \alpha_{m}, \alpha_{m+1}, \alpha_{m+2}\right) \in(\mathbb{C}-\mathbb{Z})^{m+3}, \quad \alpha_{m+2}=-\alpha_{0}-\alpha_{1}-\cdots-\alpha_{m}-\alpha_{m+1} .
$$

Let $C_{k}^{u}$ be the space of finite linear combinations $\sum_{j} a_{j} \Delta_{j} \otimes u_{\Delta_{j}}(t)$, where $a_{j} \in \mathbb{C}, \Delta_{j}$ is a $k$-simplex in $T_{x}$ and $u_{\Delta_{j}}(t)$ is a branch of $u(t)$ on $\Delta_{j}$. A twisted boundary operator is given by the linear extension of

$$
\partial_{\omega}:\left.C_{k}^{u} \ni \Delta \otimes u_{\Delta}(t) \mapsto \partial \Delta \otimes u_{\Delta}(t)\right|_{\partial \Delta} \in C_{k-1}^{u} \quad(k=0,1,2),
$$

where $\Delta$ is a $k$-simplex in $T_{x}, \partial$ is the topological boundary operator, and $\left.u_{\Delta}(t)\right|_{\partial \Delta}$ is the restriction of $u_{\Delta}(t)$ to $\partial \Delta$. A twisted homology group is defined as the quotient

$$
H_{1}\left(C_{\bullet}^{u}, \partial_{\omega}\right)=\operatorname{ker}\left(\partial_{\omega}: C_{1}^{u} \rightarrow C_{0}^{u}\right) / \partial_{\omega}\left(C_{2}^{u}\right)
$$

On the other hand, we set a single-valued rational 1-form $\omega$ on $T_{x}$ by the logarithmic exterior derivative of the multi-valued function $u(t)$ :

$$
\omega=d \log (u(t))=\frac{d u(t)}{u(t)}=\sum_{j=0}^{m+1} \frac{\alpha_{j} d t}{t-x_{j}} .
$$

A twisted cohomology group is defined as the quotient

$$
H^{1}\left(\mathcal{E}^{\bullet}, \nabla_{\omega}\right)=\operatorname{ker}\left(\nabla_{\omega}: \mathcal{E}^{1} \rightarrow \mathcal{E}^{2}\right) / \nabla_{\omega}\left(\mathcal{E}^{0}\right)
$$

by a twisted exterior derivative

$$
\nabla_{\omega}: \mathcal{E}^{k} \ni \psi \mapsto(d+\omega \wedge) \psi \in \mathcal{E}^{k+1} \quad(k=0,1,2),
$$

where $\mathcal{E}^{k}$ is the space of smooth $k$-forms on $T_{x}$. These groups are dual to each other by the pairing

$$
\langle\psi, \gamma\rangle=\sum_{i} a_{i} \int_{I_{i}} u_{I_{i}}(t) \psi,
$$


where $\psi$ is a $\nabla_{\omega}$-closed 1 -form on $T_{x}$ satisfying $\nabla_{\omega} \psi=0$ and $\gamma=\sum_{i} a_{i} I_{i} \otimes u_{J_{i}}(t)$ is a twisted 1-cycle satisfying $\partial_{\omega}(\gamma)=0$. We show that the dimensions of these groups are equal to $-\chi\left(T_{x}\right)=m+1$, where $\chi\left(T_{x}\right)$ is the Euler number of $T_{x}$.

By using $\partial_{-\omega}$ and $\nabla_{-\omega}$ given by $u^{-1}(t)=1 / u(t)$ instead of $u(t)$, we have $H_{1}\left(C_{\bullet}^{u^{-1}}, \partial_{-\omega}\right)$ and $H^{1}\left(\mathcal{E}^{\bullet}, \nabla_{-\omega}\right)$. There are an intersection form between $H_{1}\left(C_{\bullet}^{u}, \partial_{\omega}\right)$ and $H_{1}\left(C_{\bullet}^{u^{-1}}, \partial_{-\omega}\right)$, and that between $H^{1}\left(\mathcal{E}^{\bullet}, \nabla_{\omega}\right)$ and $H^{1}\left(\mathcal{E}^{\bullet}, \nabla_{-\omega}\right)$. We explain how to evaluate intersection numbers for twisted 1 -cycles in $\S 6$ and those for $\nabla_{ \pm \omega}$-closed 1-forms in $\S 8$.

It is known that the intersection forms are compatible with the natural pairings between twisted homology and cohomology groups. This compatibility yields a twisted period relation, which relates intersection numbers and period integrals regarded as some kinds of hypergeometric functions. In case of $m=0$, we have the simplest example, which is the inversion formula

$$
B(p, q) \cdot B(-p,-q)=\frac{2 \pi \sqrt{-1}(p+q)}{p q} \cdot \frac{1-e^{2 \pi \sqrt{-1}(p+q)}}{\left(1-e^{2 \pi \sqrt{-1}} p\right)\left(1-e^{2 \pi \sqrt{-1} q}\right)}
$$

for the Beta function

$$
B(p, q)=\int_{0}^{1} t^{p}(1-t)^{q} \frac{d t}{t(1-t)} \quad(\operatorname{Re}(p), \operatorname{Re}(q)>0),
$$

extended to a meromorphic function on $\mathbb{C}^{2}$ by the functional equations

$$
B(p, q)=\frac{\Gamma(p) \Gamma(q)}{\Gamma(p+q)}, \quad p \Gamma(p)=\Gamma(p+1)=\int_{0}^{\infty} e^{-t} t^{p} d t \quad(\operatorname{Re}(p)>-1) .
$$

Here $B(p, q)$ and $B(-p,-q)$ are regarded as period integrals, and the right hand side of (2) is the product of the intersection number of $\nabla_{ \pm \omega}$-closed 1-forms and that of twisted 1-cycles.

For case $m=1$, we also show that the twisted period relation yields identities among several values of the hypergeometric series such as

$$
F(a, b, c ; x) F(1-a, 1-b, 2-c ; x)=F(b-c+1, a-c+1,2-c ; x) F(c-a, c-b, c ; x) .
$$

For general $m$, we give identities among several values of the hypergeometric series $F_{D}$ by the twisted period relation.

In Appendix, we show that Elliott's identity can be obtained from the twisted period relation.

\section{Regularization of open intervals}

For the integrals (1) or (3), we need the convergence condition $\operatorname{Re}(a), \operatorname{Re}(c-a)>0$ or $\operatorname{Re}(p), \operatorname{Re}(q)>0$. Let us change these conditions into $a, c-a \notin \mathbb{Z}$ or $p, q \notin \mathbb{Z}$.

Let $C_{0}^{+\varepsilon}$ be a circle with center $t=0$, radius $\varepsilon$ and terminal $t=\varepsilon$, and $C_{1}^{-\varepsilon}$ be that with center $t=1$, radius $\varepsilon$ and terminal $t=1-\varepsilon$, where $\varepsilon$ is a sufficiently small positive number; see Figure 1.

Proposition 1. If $\operatorname{Re}(p), \operatorname{Re}(q)>0, p, q \notin \mathbb{Z}$ then

$$
B(p, q)=\frac{1}{e^{2 \pi \sqrt{-1} p}-1} \int_{C_{0}^{+\varepsilon}} u(t) \varphi+\int_{\varepsilon}^{1-\varepsilon} u(t) \varphi-\frac{1}{e^{2 \pi \sqrt{-1}} q-1} \int_{C_{1}^{-\varepsilon}} u(t) \varphi,
$$




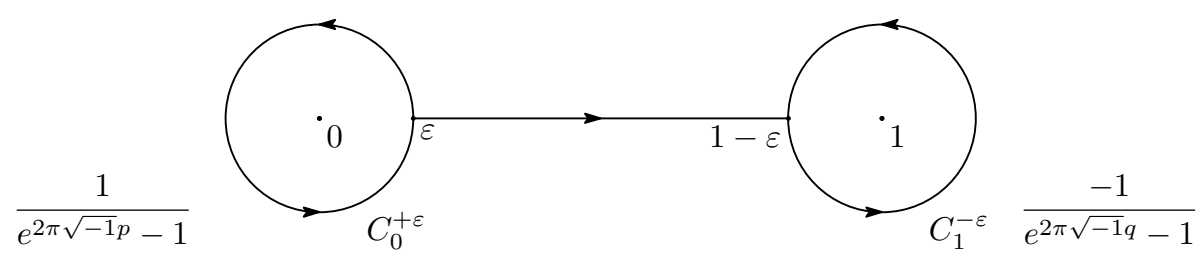

Figure 1: Regularization of the open interval $(0,1)$

where $\varphi=\frac{d t}{t(1-t)}, u(t)=t^{p}(1-t)^{q}$, its branch on $[\varepsilon, 1-\varepsilon]$ is assigned by $\arg (t)=\arg (1-t)=0$, and those on $C_{0}^{+\varepsilon}$ and $C_{1}^{-\varepsilon}$ are assigned by the $\arg (t)=\arg (1-t)=0$ at their start points.

Proof. We show that the right hand side of (4) is independent of $\varepsilon$. This property yields the identity, since

$$
\lim _{\varepsilon \rightarrow 0} \int_{C_{0}^{+\varepsilon}} u(t) \varphi=\lim _{\varepsilon \rightarrow 0} \int_{C_{1}^{-\varepsilon}} u(t) \varphi=0
$$

and

$$
\lim _{\varepsilon \rightarrow 0} \int_{\varepsilon}^{1-\varepsilon} u(t) \varphi=B(p, q)
$$

under $\operatorname{Re}(p), \operatorname{Re}(q)>0$. Take a circle $C_{0}^{+\delta}$ for $0<\delta<\varepsilon$, and consider the difference

$$
\frac{1}{e^{2 \pi \sqrt{-1} p}-1}\left[\int_{C_{0}^{+\varepsilon}} u(t) \varphi-\int_{C_{0}^{+\delta}} u(t) \varphi\right]-\int_{\delta}^{\varepsilon} u(t) \varphi .
$$

It is equal to

$$
\frac{1}{e^{2 \pi \sqrt{-1} p}-1} \int_{C} t^{p}(1-t)^{q} \frac{d t}{t(1-t)},
$$

which vanishes by Cauchy's integral theorem, where the path $C$ is in Figure 2. We can similarly

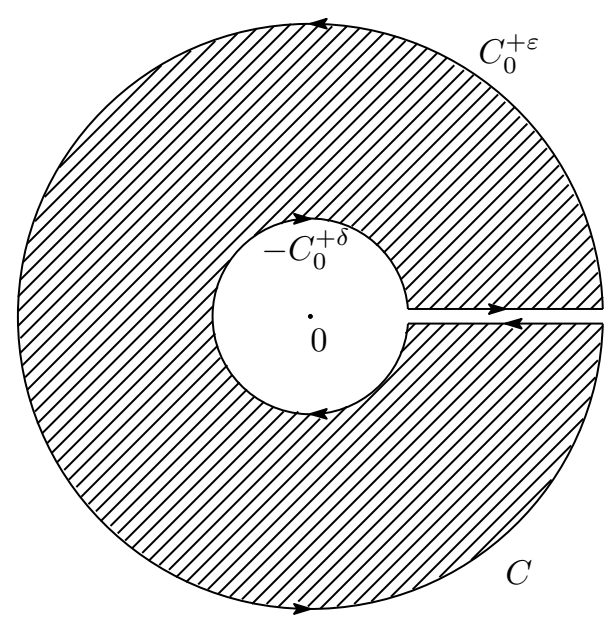

Figure 2: Path $C$

show that the right hand side of (4) is independent of $\varepsilon$ for the circle $C_{1}^{-\varepsilon}$. 
Definition 1 ([AK, Example 2.1]). We define the regularization of the open interval $(0,1)$ with respect to $u(t)=t^{p}(1-t)^{q}$ by the formal sum

$$
\frac{1}{e^{2 \pi \sqrt{-1} p}-1} C_{0}^{+\varepsilon}+[\varepsilon, 1-\varepsilon]-\frac{1}{e^{2 \pi \sqrt{-1} q}-1} C_{1}^{-\varepsilon}
$$

with assignments of branches of $u(t)$ on $C_{0}^{+\varepsilon},[\varepsilon, 1-\varepsilon], C_{1}^{-\varepsilon}$ as in Proposition 1 .

Note that each path integral of (4) in Proposition 1 reduces to an integral of a continuous function over a bounded closed interval. Since it is well defined under the condition $p, q \notin \mathbb{Z}$, we remove the convergence condition $\operatorname{Re}(p), \operatorname{Re}(q)>0$ for implicit integrals for $B(p, q)$.

We can apply this cycle to the Euler type integral (1) for hypergeometric series. We change the convergence condition $\operatorname{Re}(a), \operatorname{Re}(c-a)>0$ into $a, c-a \notin \mathbb{Z}$ by using this cycle. To prove (1) integrated along this cycle, we use the Taylor expansion

$$
(1-t x)^{-b}=\sum_{n=0}^{\infty} \frac{(b)_{n}}{n !} t^{n} x^{n}
$$

and change the order of the infinite series and the integral. This order change is permitted under the uniform convergence of the series for the fixed variable $x \in \mathbb{D}$. Note that we need much more strong conditions for changing the order of an infinite series and an implicit integral.

Moreover, we will see an advantage to be able to define the intersection form by introducing the regularization of open intervals.

\section{Lauricella's hypergeometric system $\mathcal{F}_{D}$}

In this section, we generalize the Euler type integral (1) of $F(a, b, c ; x)$ or (3) for $B(p, q)$ by referring to [Y1, §6]. By the variable change $s=1 / t,(1)$ and (3) are transformed into

$$
\int_{1}^{\infty} s^{b-c}(s-x)^{-b}(s-1)^{c-a} \frac{d s}{s-1}, \quad \int_{1}^{\infty} s^{-p-q}(s-1)^{q} \frac{d s}{s-1} .
$$

Here we generalize $u(t)$ to

$$
u(t)=u(t, x)=\prod_{j=0}^{m+2}\left(t-x_{j}\right)^{\alpha_{j}}=t^{\alpha_{0}}\left[\prod_{j=1}^{m}\left(t-x_{j}\right)^{\alpha_{j}}\right](t-1)^{\alpha_{m+1}},
$$

where $x_{0}=0, x_{1}, \ldots, x_{m}, x_{m+1}=1, x_{m+2}=\infty$ are mutually different complex variables, and $\alpha_{j}$ are complex parameters satisfying

$$
\alpha_{0}, \alpha_{1}, \ldots, \alpha_{m}, \alpha_{m+1}, \alpha_{m+2} \notin \mathbb{Z}, \quad \sum_{j=0}^{m+2} \alpha_{j}=0 .
$$

We set

$$
\alpha=\left(\alpha_{0}, \alpha_{1}, \ldots, \alpha_{m}, \alpha_{m+1}, \alpha_{m+2}\right)
$$

and assume (6) throughout this article unless otherwise specified. We consider the integral

$$
\int_{1}^{\infty} u(t) \varphi, \quad \varphi=\frac{d t}{t-1}
$$


which converges under our assumption (6) if we use the regularization of $(1, \infty)$ with respect to $u(t)$. Introduce parameters $a, b=\left(b_{1}, \ldots, b_{m}\right), c$ from $\alpha$ as

$$
a=\alpha_{m+2}, \quad b=\left(-\alpha_{1}, \ldots,-\alpha_{m}\right), \quad c=\alpha_{m+1}+\alpha_{m+2} .
$$

In case of $m=0$, the integral (7) is equal to $B\left(\alpha_{1}, \alpha_{2}\right)=B(a, c-a)$; in case of $m=1$, it is equal to

$$
B\left(\alpha_{2}, \alpha_{3}\right) F\left(\alpha_{3},-\alpha_{1}, \alpha_{2}+\alpha_{3} ; x_{1}\right)=B(a, c-a) F\left(a, b, c ; x_{1}\right)
$$

for $c=\alpha_{2}+\alpha_{3} \neq 0,-1,-2, \ldots$ and $x_{1} \in \mathbb{D}$. For general $m$, if $c=\alpha_{m+1}+\alpha_{m+2}$ is different from $0,-1 .-2, \ldots$ and $x=\left(x_{1}, \ldots, x_{m}\right)$ belongs to

$$
\mathbb{D}_{m}=\left\{x \in \mathbb{C}^{m}\left|\max _{1 \leq j \leq m}\right| x_{j} \mid<1\right\}
$$

then the integral (7) can be expressed as

$$
B\left(\alpha_{m+1}, \alpha_{m+2}\right) F_{D}\left(\alpha_{m+2},-\alpha_{1}, \ldots,-\alpha_{m}, \alpha_{m+1}+\alpha_{m+2} ; x\right)=B(c-a, a) F_{D}(a, b, c ; x),
$$

where $F_{D}(a, b, c ; x)$ is Lauricella's hypergeometric series defined by

$$
F_{D}(a, b, c ; x)=\sum_{n \in \mathbb{N}_{0}^{m}} \frac{(a)_{n_{1}+\cdots+n_{m}} \prod_{j=1}^{m}\left(b_{j}\right)_{n_{j}}}{(c)_{n_{1}+\cdots+n_{m}} \prod_{j=1}^{m} n_{j} !} \prod_{j=1}^{m} x_{j}^{n_{j}} .
$$

The differential operators

$$
\begin{aligned}
& x_{j}\left(1-x_{j}\right) \partial_{j}^{2}+\left(1-x_{j}\right) \sum_{1 \leq k \leq m}^{k \neq j} x_{k} \partial_{j} \partial_{k}+\left[c-\left(a+b_{j}+1\right) x_{j}\right] \partial_{j}-b_{j} \sum_{1 \leq k \leq m}^{k \neq j} x_{k} \partial_{k}-a b_{j},(1 \leq j \leq m) \\
& \left(x_{j}-x_{k}\right) \partial_{j} \partial_{k}-b_{k} \partial_{j}+b_{j} \partial_{k}, \quad(1 \leq j<k \leq m)
\end{aligned}
$$

annihilate the series $F_{D}(a, b, c ; x)$, where $\partial_{j}=\frac{\partial}{\partial x_{j}}(1 \leq j \leq m)$. The partial differential equations given by their actions generate Lauricella's hypergeometric system $\mathcal{F}_{D}(a, b, c)$, which is a holonomic system of rank $m+1$ with regular locus

$$
X=\left\{x \in \mathbb{C}^{m} \mid \prod_{j=1}^{m}\left[x_{j}\left(1-x_{j}\right)\right] \prod_{1 \leq j<k \leq m}\left(x_{j}-x_{k}\right) \neq 0\right\} .
$$

This means that the vector space of solutions to $\mathcal{F}_{D}(a, b, c)$ on a small neighborhood of any $x \in X$ is $m+1$ dimensional.

\section{Twisted Stokes' Theorem}

We consider the treatment of a multi-valued 1-form $u(t) \varphi(t)$ in (7) by referring to [AK, §2.1]. Let $\psi$ be a smooth $k$-form on $T_{x}=\mathbb{P}^{1}-\left\{x_{0}, x_{1}, \ldots, x_{m+2}\right\}=\mathbb{C}-\left\{0, x_{1}, \ldots, x_{m}, 1\right\}$, and let $\Delta$ be a $k$-simplex in $T_{x}$. To define an integral

$$
\int_{\Delta} u(t) \psi
$$


we need to assign a branch $u_{\Delta}(t)$ of $u(t)$. Though $u(t)$ is multi valued on $T_{x}$, the restriction of any branch of $u(t)$ to $\Delta$ is single valued since $\Delta$ is simply connected. Thus we separate the multi-valued function $u(t)$ and $\psi$ from $u(t) \psi$, and regard the integral (9) with assignment of a branch $u_{\Delta}(t)$ of $u(t)$ on $\Delta$ as a pairing $\left\langle\psi, \Delta \otimes u_{\Delta}(t)\right\rangle$ between $\psi$ and the pair $\Delta \otimes u_{\Delta}(t)$ of $\Delta$ and $u_{\Delta}(t)$.

We rewrite Stokes' theorem

$$
\int_{D} d\left(u_{D}(t) \psi\right)=\int_{\partial D} u_{D}(t) \psi
$$

under this policy, where $\psi$ is a smooth $k$-form, $D$ is a $(k+1)$-simplex, $u_{D}(t)$ is a branch of $u(t)$ and $\partial$ is the boundary operator. The left hand side of (10) is

$$
d\left(u_{D}(t) \psi\right)=u_{D}(t) d \psi+d u_{D}(t) \wedge \psi=u_{D}(t)(d \psi+\omega \wedge \psi)=u_{D}(t) \nabla_{\omega} \psi,
$$

where we set

$$
\omega=d \log \left(u_{D}(t)\right)=\frac{d u_{D}(t)}{u_{D}(t)}=\sum_{j=0}^{m+1} \frac{\alpha_{j} d t}{t-x_{j}},
$$

and introduce a twisted exterior derivative

$$
\nabla_{\omega}=d+\omega \wedge .
$$

Here note that $\omega=d \log u_{D}(t)=d \log u(t)$ is a single-valued smooth 1-form on $T_{x}$ though $u(t)$ is multi valued on $T_{x}$.

On the other hand, the right hand side of $(10)$ is $\left\langle\psi,\left.(\partial D) \otimes u_{D}(t)\right|_{\partial D}\right\rangle$ under this policy, where $\left.u_{D}(t)\right|_{\partial D}$ is the restriction of $u_{D}(t)$ to $\partial D$. Thus we define a twisted boundary operator $\partial_{\omega}$ by

$$
\partial_{\omega}\left(D \otimes u_{D}(t)\right)=\left.(\partial D) \otimes u_{D}(t)\right|_{\partial D} .
$$

We summarize these results as follows.

Theorem 1 (Twisted Stokes’ Theorem ([AK, §2.1.2,2.1.3])).

$$
\left\langle\nabla_{\omega} \psi, D \otimes u_{D}(t)\right\rangle=\left\langle\psi, \partial_{\omega}\left(D \otimes u_{D}(t)\right)\right\rangle .
$$

Let $\psi$ be a smooth $k$-form $\psi$ on $T_{x}$. If $\nabla_{\omega} \psi=0$ then $\psi$ is said to be $\nabla_{\omega}$-closed, and if there exists a smooth $(k-1)$-form $f$ on $T_{x}$ such that $\nabla_{\omega} f=\psi$, then $\psi$ is said to be $\nabla_{\omega}$-exact. Since $\nabla_{\omega} \circ \nabla_{\omega}=0$, if $\psi$ is $\nabla_{\omega}$-exact then $\psi$ is $\nabla_{\omega}$-closed. Any holomorphic 1-form $\varphi$ on $T_{x}$ is $\nabla_{\omega}$-closed since $\nabla_{\omega}(\varphi)=d \varphi+\omega \wedge \varphi=0$ by $d t \wedge d t=0$. The 1 -form $\omega$ is $\nabla_{\omega}$-exact since $\omega=\nabla_{\omega} 1$.

A finite linear combination

$$
\gamma=\sum_{j} a_{j} \Delta_{j} \otimes u_{\Delta_{j}}(t)
$$

is said to be a twisted $k$-chain, where $a_{j} \in \mathbb{C}$, each $\Delta_{j} \otimes u_{\Delta_{j}}(t)$ is a pair of a $k$-simplex $\Delta_{j}$ in $T_{x}$ and a branch $u_{\Delta_{j}}(t)$ of $u(t)$ on $\Delta_{j}$. If $\partial_{\omega}(\gamma)=0$ then $\gamma$ is said to be a twisted $k$-cycle, where the twisted boundary operator $\partial_{\omega}$ is extended linearly. Since $\partial_{\omega} \circ \partial_{\omega}=0, \partial_{\omega}(\gamma)$ is a twisted $(k-1)$-cycle for any twisted $k$-chain $\gamma$.

In case of $m=0$, we define a twisted 1-chain as

$$
\gamma_{0}=\frac{1}{e^{2 \pi \sqrt{-1}} p-1} C_{0}^{+\varepsilon} \otimes u_{0}(t)+[\varepsilon, 1-\varepsilon] \otimes u_{I}(t)-\frac{1}{e^{2 \pi \sqrt{-1} q}-1} C_{1}^{-\varepsilon} \otimes u_{1}(t)
$$


by $I=[\varepsilon, 1-\varepsilon], C_{0}^{+\varepsilon}, C_{1}^{-\varepsilon}$ and the branches $u_{I}(t), u_{0}(t), u_{1}(t)$ of $u(t)=t^{p}(1-t)^{q}$ on them as in $\S 2$. (Strictly speaking, we should divide $C_{0}^{+\varepsilon}$ and $C_{1}^{-\varepsilon}$ into upper and lower semi-circles, since $C_{0}^{+\varepsilon}$ and $C_{1}^{-\varepsilon}$ are not isomorphic to a 1 -simplex in $T_{x}$.) It is a twisted 1-cycle since

$$
\begin{aligned}
\partial_{\omega}\left(\gamma_{0}\right)= & \frac{[t]_{t=\varepsilon} \otimes e^{2 \pi \sqrt{-1}} p u_{0}(\varepsilon)-[t]_{t=\varepsilon} \otimes u_{0}(\varepsilon)}{e^{2 \pi \sqrt{-1}} p-1}+\left([t]_{t=1-\varepsilon} \otimes u_{I}(1-\varepsilon)-[t]_{t=\varepsilon} \otimes u_{I}(\varepsilon)\right) \\
& -\frac{[t]_{t=1-\varepsilon} \otimes e^{2 \pi \sqrt{-1}} u_{1}(1-\varepsilon)-[t]_{t=1-\varepsilon} \otimes u_{1}(1-\varepsilon)}{e^{2 \pi \sqrt{-1} q}-1} \\
= & 0,
\end{aligned}
$$

where $u_{0}(\varepsilon)$ and $u_{1}(1-\varepsilon)$ denote the values of $u_{0}(t)$ and $u_{1}(t)$ at the start points of $C_{0}^{+\varepsilon}$ and $C_{1}^{-\varepsilon}$, respectively. Here note that the values of $u_{0}(t)$ and $u_{1}(t)$ at the end points of $C_{0}^{+\varepsilon}$ and $C_{1}^{-\varepsilon}$ are equal to $e^{2 \pi \sqrt{-1}} p u_{0}(\varepsilon)$ and $e^{2 \pi \sqrt{-1}} q u_{1}(1-\varepsilon)$, respectively, and that

$$
u_{0}(\varepsilon)=u_{I}(\varepsilon), \quad u_{1}(1-\varepsilon)=u_{I}(1-\varepsilon) .
$$

We can regard the integral (1) or (3) as a pairing between a $\nabla_{\omega}$-closed 1-form and a twisted 1-cycle.

Remark 1. Since the open interval $(0,1)$ in $T_{x}=\mathbb{C}-\{0,1\}$ cannot be expressed by a finite sum of 1-simplexes in $T_{x}$, we cannot regard the pair $(0,1) \otimes u_{(0,1)}(t)$ of $(0,1)$ and a branch $u_{(0,1)}(t)$ of $u(t)$ as a twisted 1-chain for this definition. To regard $(0,1) \otimes u_{(0,1)}(t)$ as a twisted 1-chain, we need to extend "finite sums" to "infinite sums with local finiteness", which are not treated in this article.

\section{Twisted homology groups}

The vector space of twisted $k$-chains in $T_{x}$ is denoted by $C_{k}^{u}$. Let $\varphi$ be a $\nabla_{\omega}$-closed 1 -form on $T_{x}$, and $\gamma$ be a twisted 1-cycle in $T_{x}$. Since

$$
\left\langle\varphi, \partial_{\omega}(G)\right\rangle=\left\langle\nabla_{\omega} \varphi, G\right\rangle=\langle 0, G\rangle=0,
$$

for any $G \in C_{k}^{u}$ by Twisted Stocks' theorem, we have

$$
\left\langle\varphi, \gamma+\partial_{\omega}(G)\right\rangle=\langle\varphi, \gamma\rangle
$$

This means that $\partial_{\omega}\left(C_{2}^{u}\right)$ has no effect on the pairing $\langle\varphi, \gamma\rangle$ between a $\nabla_{\omega}$-closed 1-form $\varphi$ and a twisted 1-cycle $\gamma$ in $T_{x}$.

Definition 2 (Twisted homology group). The $k$-th twisted homology group $H_{k}\left(C_{\bullet}^{u}, \partial_{\omega}\right)$ is defined by

$$
H_{k}\left(C_{\bullet}^{u}, \partial_{\omega}\right)=\operatorname{ker}\left(\partial_{\omega}: C_{k}^{u} \rightarrow C_{k-1}^{u}\right) / \partial_{\omega}\left(C_{k+1}^{u}\right) \quad(k=0,1,2) .
$$

Proposition 2 (Dimension formula [AK, Lemma 2.14]). Under our assumption (6) on $\alpha$, we have

$$
\operatorname{dim} H_{1}\left(C_{\bullet}^{u}, \partial_{\omega}\right)=m+1 .
$$


Proof. Since $C_{-1}^{u}$ and $C_{3}^{u}$ are isomorphic to the zero vector space, we have

$$
H_{0}\left(C_{\bullet}^{u}, \partial_{\omega}\right)=C_{0}^{u} / \partial_{\omega}\left(C_{1}^{u}\right), \quad H_{2}\left(C_{\bullet}^{u}, \partial_{\omega}\right)=\operatorname{ker}\left(\partial_{\omega}: C_{2}^{u} \rightarrow C_{1}^{u}\right)
$$

Let $p$ be any point in $T_{x}$ and $C$ be a loop in $T_{x}$ with terminal $p$ turning around $x_{0}$ once positively. Then

$$
\partial_{w}\left(C \otimes u_{C}(t)\right)=\left(e^{2 \pi \sqrt{-1} \alpha_{0}}-1\right) \cdot[t]_{t=p} \otimes u_{C}(p),
$$

where $u_{C}(p)$ is the value of a branch $u_{C}(t)$ of $u(t)$ at the start point. Thus we have $C_{0}^{u}=\partial_{\omega}\left(C_{1}^{u}\right)$ which means $H_{0}\left(C_{\bullet}^{u}, \partial_{\omega}\right)=0$ under the condition $\alpha_{0} \notin \mathbb{Z}$. Since any twisted 2-chain consists of finite sum of $c_{j} \Delta_{j} \otimes u(t)$ for $c_{j} \in \mathbb{C}$ and 2-simplexes $\Delta_{j}$, we cannot eliminate their boundaries. Thus we have $\operatorname{ker}\left(\partial_{\omega}: C_{2}^{u} \rightarrow C_{1}^{u}\right)=0$, which means $H_{2}\left(C_{\bullet}^{u}, \partial_{\omega}\right)=0$.

We use the fact

$$
\chi\left(T_{x}\right)=\operatorname{dim} H_{0}\left(C_{\bullet}^{u}, \partial_{\omega}\right)-\operatorname{dim} H_{1}\left(C_{\bullet}^{u}, \partial_{\omega}\right)+\operatorname{dim} H_{2}\left(C_{\bullet}^{u}, \partial_{\omega}\right),
$$

where $\chi\left(T_{x}\right)$ is the Euler number of $T_{x}$. Since

$$
\chi\left(T_{x}\right)=\chi\left(\mathbb{P}^{1}\right)-\#\left\{x_{0}, \ldots, x_{m+2}\right\}=2-(m+3)=-m-1,
$$

we have $\operatorname{dim} H_{1}\left(C_{\bullet}^{u}, \partial_{\omega}\right)=m+1$.

Remark 2. This dimension formula is valid under the condition $\alpha \notin \mathbb{Z}^{m+3}$. If $\alpha_{j} \in \mathbb{Z}$ then we cannot use the regularization of cycles for paths with terminal $x_{j}$. For example, we give some cases $(p, q,-p-q) \notin \mathbb{Z}^{3}$ for $B(p, q): T_{x}=\mathbb{P}^{1}-\{0,1, \infty\}, u(t)=t^{p}(1-t)^{q}$ and $\operatorname{dim} H_{1}\left(C_{\bullet}^{u}, \partial_{\omega}\right)=1$.

If $p \in \mathbb{Z}$ and $q, p+q \notin \mathbb{Z}$, then the regularization of $(0,1)$ with respect to $u(t)$ cannot be defined. In this case, $C_{0}^{\varepsilon} \otimes u_{0}(t)$ becomes a twisted 1-cycle, and it spans $H_{1}\left(C_{\bullet}^{u}, \partial_{\omega}\right)$. Note that this twisted 1-cycle can be regarded as $\partial_{\omega}\left(\Delta_{0} \otimes u_{\Delta_{0}}(t)\right)$ if we permit infinite sums with locally finiteness, where $\Delta_{0}=\{t \in \mathbb{C}|0<| t \mid \leq \varepsilon\}$ and $u_{\Delta_{0}}(t)$ is the continuation of $u_{0}(t)$ to $\Delta_{0}$.

If $p, q \notin \mathbb{Z}$ and $p+q \in \mathbb{Z}$ then we have the regularization of $(0,1)$ with respect to $u(t)$, and it gives a non-zero element of $H_{1}\left(C_{\bullet}^{u}, \partial_{\omega}\right)$. In this case, if we permit infinite sums with locally finiteness then $(0,1) \otimes u_{(0,1)}(t)$ can be regarded as a twisted 1-cycle and it coincides with $\partial_{\omega}\left(\Delta_{I} \otimes u_{\Delta_{I}}(t)\right)$ up to a non-zero constant, where $\Delta_{I}=T_{x}-(0,1)$ and a branch $u_{\Delta_{I}}(t)$ of $u(t)$ is assigned by $\arg (t), \arg (1-t) \in(0,2 \pi)$ on $\Delta_{I}$.

\section{Intersection form between twisted homology groups}

By using $1 / u(t)=u^{-1}(t)$ instead of $\mathrm{u}(\mathrm{t})$, we define the spaces $C_{k}^{u^{-1}}$ of twisted $k$-chains, the twisted boundary operator $\partial_{-\omega}$ and the twisted homology groups $H_{k}\left(C_{\bullet}^{u^{-1}}, \partial_{-\omega}\right)(k=0,1,2)$.

Definition 3 (Intersection number of twisted 1-cycles). Let $\gamma=\sum_{i} a_{i} I_{i} \otimes u_{I_{i}}(t)$ and $\delta=\sum_{j} b_{j} J_{j} \otimes$ $u_{J_{j}}^{-1}(t)$ be twisted cycles, where $a_{i}, b_{j} \in \mathbb{C}, I_{i}$ and $J_{j}$ are 1-simplexes in $T_{x}$. Suppose that any $I_{i}$ and $J_{j}$ intersect transversally at every intersection point $p$ of them. The intersection number of $\gamma$ and $\delta$ is defined by

$$
\langle\gamma, \delta\rangle=\sum_{i, j} a_{i} b_{j} \sum_{p \in I_{i} \cap J_{j}}\left\langle I_{i}, J_{j}\right\rangle_{p} \cdot\left[u_{I_{i}}(p)\right] \cdot\left[u_{J_{j}}^{-1}(p)\right],
$$

where $\left\langle I_{i}, J_{j}\right\rangle_{p}$ denotes the topological intersection number of $I_{i}$ and $J_{i}$ at $p$. 
As in [KY1, §1], we have the following theorems.

Theorem 2. The intersection number (12) induces a bi-linear form between the spaces of twisted cycles $\operatorname{ker}\left(\partial_{\omega}: C_{1}^{u} \rightarrow C_{0}^{u}\right)$ and $\operatorname{ker}\left(\partial_{-\omega}: C_{1}^{u^{-1}} \rightarrow C_{0}^{u^{-1}}\right)$, which descends to that between $H_{1}\left(C_{\bullet}^{u}, \partial_{\omega}\right)$ and $H_{1}\left(C_{\bullet}^{u^{-1}}, \partial_{-\omega}\right)$. It is non-degenerate.

Theorem 3 ([AK, §2.3.3], [KY1, Theorem 2.1] and [Y2, §4.7]). Suppose that $x_{1}, \ldots, x_{m} \in \mathbb{R}$, $0=x_{0}<x_{1}<\cdots<x_{m}<x_{m+1}=1, x_{m+2}=\infty$. Let $\gamma_{j}$ be the twisted cycle given by the regularization of $I_{j}=\left(x_{j}, x_{j+1}\right)$ with respect to $u(t)$, and let $\delta_{k}$ be that of $I_{k}=\left(x_{k}, x_{k+1}\right)$ with respect to $u^{-1}(t)$. Then we have

$$
\left\langle\gamma_{j}, \delta_{k}\right\rangle=\left\{\begin{array}{cl}
\frac{-\theta_{j}}{1-\theta_{j}} & \text { if } k=j-1, \\
\frac{1-\theta_{j} \theta_{j+1}}{\left(1-\theta_{j}\right)\left(1-\theta_{j+1}\right)} & \text { if } k=j, \\
\frac{-1}{1-\theta_{j+1}} & \text { if } k=j+1, \\
0 & \text { otherwise, }
\end{array}\right.
$$

where $\theta_{j}=\exp \left(2 \pi \sqrt{-1} \alpha_{j}\right)$.

Proof. Let us compute $\left\langle\gamma_{j}, \delta_{j}\right\rangle$. We can regard the branches of $u(t)$ and $u^{-1}(t)$ on any components of $\gamma_{j}$ and $\delta_{j}$ as the analytic continuations of branches $u_{\mathbb{H}}(t)$ and $u_{\mathbb{H}}^{-1}(t)$ defined on the upper half space of $T_{x}$ satisfying $u_{\mathbb{H}}(t) \cdot u_{\mathbb{H}}^{-1}(t)=1$. These continuations are denoted by $u_{\gamma_{j}}(t)$ and $u_{\delta_{j}}^{-1}(t)$. We can see by Figure 3 that the topological intersection number of the 1-simplexes of $\gamma_{j}$ and $\delta_{j}$ at $p_{1}$ is -1 , that at $p_{2}$ is +1 , and

$$
u_{\gamma_{j}}\left(p_{1}\right) \cdot u_{\delta_{j}}^{-1}\left(p_{1}\right)=u_{\mathbb{H}}\left(p_{1}\right) \cdot u_{\mathbb{H}}^{-1}\left(p_{1}\right)=1, \quad u_{\gamma_{j}}\left(p_{2}\right) \cdot u_{\delta_{j}}^{-1}\left(p_{2}\right)=\theta_{j+1} u_{\mathbb{H}}\left(p_{2}\right) \cdot u_{\mathbb{H}}^{-1}\left(p_{2}\right)=\theta_{j+1} .
$$

Here note that the value of $u_{\gamma_{j}}(t)$ at $p_{2}$ is multiplied $\theta_{j+1}$ to $u_{\mathbb{H}}(t)$ since the variable $t$ turns once around $x_{j+1}$ positively along the circle. Thus the intersection number $\left\langle\gamma_{j}, \delta_{j}\right\rangle$ is

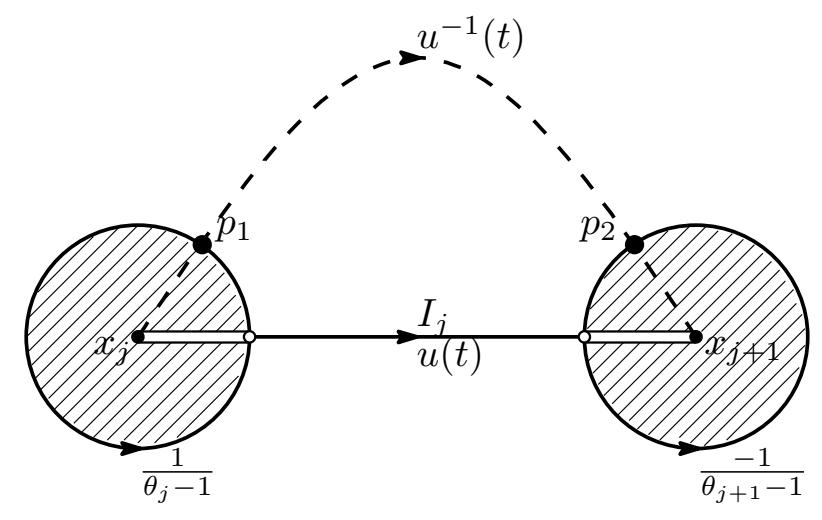

Figure 3: Intersection of twisted cycles

$$
(-1) \frac{1}{\theta_{j}-1}+\theta_{j+1} \frac{-1}{\theta_{j+1}-1}=\frac{1-\theta_{j} \theta_{j+1}}{\left(1-\theta_{j}\right)\left(1-\theta_{j+1}\right)}
$$


We can similarly compute other $\left\langle\gamma_{j}, \delta_{k}\right\rangle$.

We can show that the intersection form between $H_{1}\left(C_{\bullet}^{u}, \partial_{\omega}\right)$ and $H_{1}\left(C_{\bullet}^{u^{-1}}, \partial_{-\omega}\right)$ is nondegenerate by the regularity of the intersection matrix

$$
H_{h}=\left(\left\langle\gamma_{j}, \delta_{k}\right\rangle\right)_{0 \leq j, k \leq m}=\left(\begin{array}{ccccc}
\frac{1-\theta_{0} \theta_{1}}{\left(1-\theta_{0}\right)\left(1-\theta_{1}\right)} & \frac{-1}{1-\theta_{1}} & 0 & \cdots & 0 \\
\frac{-\theta_{1}}{1-\theta_{1}} & \frac{1-\theta_{1} \theta_{2}}{\left(1-\theta_{1}\right)\left(1-\theta_{2}\right)} & \frac{-1}{1-\theta_{2}} & \ddots & \\
0 & \frac{-\theta_{2}}{1-\theta_{2}} & \frac{1-\theta_{2} \theta_{3}}{\left(1-\theta_{2}\right)\left(1-\theta_{3}\right)} & \ddots & \vdots \\
\vdots & \ddots & \ddots & \ddots & \\
0 & & \cdots & & \frac{1-\theta_{m} \theta_{m+1}}{\left(1-\theta_{m}\right)\left(1-\theta_{m+1}\right)}
\end{array}\right) .
$$

Under our assumption (6), $\operatorname{det}\left(H_{h}\right)=\frac{1-\theta_{m+2}^{-1}}{\left(1-\theta_{0}\right)\left(1-\theta_{1}\right) \cdots\left(1-\theta_{m+1}\right)}$ does not vanish.

By Theorem 3, the intersection number between twisted cycles defining $B(p, q)$ and $B(-p,-q)$ is

$$
\frac{1-e^{2 \pi \sqrt{-1}(p+q)}}{\left(1-e^{2 \pi \sqrt{-1}} p\right)\left(1-e^{2 \pi \sqrt{-1} q}\right)} .
$$

\section{Twisted cohomology groups}

Recall that Twisted Stokes' Theorem

$$
\left\langle\nabla_{\omega} \psi, D \otimes u(t)\right\rangle=\left\langle\psi, \partial_{\omega}(D \otimes u(t))\right\rangle,
$$

where $\omega=d \log u(t), \nabla_{\omega}=d+\omega \wedge, \psi$ is a smooth $k$-form, $D$ is a $(k+1)$-chain in $T_{x}$. Let $\varphi$ be a $\nabla_{\omega}$-closed 1-form on $T_{x}$, and $\gamma$ be a twisted 1-cycle in $T_{x}$. Since

$$
\left\langle\nabla_{\omega} f, \gamma\right\rangle=\left\langle f, \partial_{\omega} \gamma\right\rangle=\langle f, 0\rangle=0
$$

for any smooth function $f$ on $T_{x}$ by Twisted Stocks' theorem, we have

$$
\left\langle\varphi+\nabla_{\omega} f, \gamma\right\rangle=\langle\varphi, \gamma\rangle
$$

It means that $\nabla_{\omega}\left(\mathcal{E}^{0}\right)$ has no effect on the pairing $\langle\varphi, \gamma\rangle$ between a $\nabla_{\omega}$-closed 1-form $\varphi$ and a twisted 1-cycle $\gamma$ in $T_{x}$, where $\mathcal{E}^{0}$ is the space of smooth functions on $T_{x}$.

Let $\mathcal{E}^{k}$ and $\mathcal{E}_{c}^{k}$ be the space of smooth $k$-forms on $T_{x}$ and that with compact support. Note that

$$
\mathcal{E}_{c}^{k} \subset \mathcal{E}^{k}, \quad \frac{d t}{t(1-t)}, \frac{d t}{t-1} \in \mathcal{E}^{1}-\mathcal{E}_{c}^{1},
$$

and that $\psi \in \mathcal{E}^{k}$ belongs to $\mathcal{E}_{c}^{k}$ if and only if $\psi \equiv 0$ on a small neighborhood $U_{j}$ of $x_{j}(j=$ $0,1, \ldots, m+2)$.

Definition 4 (Twisted cohomology groups). A twisted cohomology group and that with compact support are defined as

$$
\begin{aligned}
& H^{k}\left(\mathcal{E}^{\bullet}, \nabla_{\omega}\right)=\operatorname{ker}\left(\nabla_{\omega}: \mathcal{E}^{k} \rightarrow \mathcal{E}^{k+1}\right) / \nabla_{\omega}\left(\mathcal{E}^{k-1}\right), \\
& H^{k}\left(\mathcal{E}_{c}^{\bullet}, \nabla_{\omega}\right)=\operatorname{ker}\left(\nabla_{\omega}: \mathcal{E}_{c}^{k} \rightarrow \mathcal{E}_{c}^{k+1}\right) / \nabla_{\omega}\left(\mathcal{E}_{c}^{k-1}\right),
\end{aligned}
$$

for $k=0,1,2$. 
By regarding $\psi \in \mathcal{E}_{c}^{1}$ as an element of $\mathcal{E}^{1}$, we have a natural map

$$
H^{1}\left(\mathcal{E}_{c}^{\bullet}, \nabla_{\omega}\right) \rightarrow H^{1}\left(\mathcal{E}^{\bullet}, \nabla_{\omega}\right) .
$$

Proposition 3 (Dimension formula [KN, Main Theorem, Remark 2]). Under our assumption (6) on $\alpha$, we have

$$
\operatorname{dim} H^{1}\left(\mathcal{E}^{\bullet}, \nabla_{\omega}\right)=\operatorname{dim} H^{1}\left(\mathcal{E}_{c}^{\bullet}, \nabla_{\omega}\right)=m+1,
$$

and the natural map (13) is an isomorphism.

Proof. To obtain the claim for the dimension, we show that the spaces

$$
\begin{array}{ll}
H^{0}\left(\mathcal{E}^{\bullet}, \nabla_{\omega}\right)=\operatorname{ker}\left(\nabla_{\omega}: \mathcal{E}^{0} \rightarrow \mathcal{E}^{1}\right), & H^{2}\left(\mathcal{E}^{\bullet}, \nabla_{\omega}\right)=\mathcal{E}^{2} / \nabla_{\omega}\left(\mathcal{E}^{1}\right), \\
H_{c}^{0}\left(\mathcal{E}^{\bullet}, \nabla_{\omega}\right)=\operatorname{ker}\left(\nabla_{\omega}: \mathcal{E}_{c}^{0} \rightarrow \mathcal{E}_{c}^{1}\right), & H_{c}^{2}\left(\mathcal{E}^{\bullet}, \nabla_{\omega}\right)=\mathcal{E}_{c}^{2} / \nabla_{\omega}\left(\mathcal{E}_{c}^{1}\right),
\end{array}
$$

vanish, and use the fact

$$
\begin{aligned}
\chi\left(T_{x}\right) & =\operatorname{dim} H^{0}\left(\mathcal{E}^{\bullet}, \nabla_{\omega}\right)-\operatorname{dim} H^{1}\left(\mathcal{E}^{\bullet}, \nabla_{\omega}\right)+\operatorname{dim} H^{2}\left(\mathcal{E}^{\bullet}, \nabla_{\omega}\right) \\
& =\operatorname{dim} H^{0}\left(\mathcal{E}_{c}^{\bullet}, \nabla_{\omega}\right)-\operatorname{dim} H^{1}\left(\mathcal{E}_{c}^{\bullet}, \nabla_{\omega}\right)+\operatorname{dim} H^{2}\left(\mathcal{E}_{c}^{\bullet}, \nabla_{\omega}\right) .
\end{aligned}
$$

Let us show $H^{0}\left(\mathcal{E}^{\bullet}, \nabla_{\omega}\right)=H_{c}^{0}\left(\mathcal{E}^{\bullet}, \nabla_{\omega}\right)=0$. Regard $\nabla_{\omega} f=0$ as a first order linear differential equation for unknown function $f$. Its solution is $c / u(t)(c \in \mathbb{C})$ in a neighborhood of any $t \in T_{x}$. In fact,

$$
\nabla_{\omega} \frac{1}{u(t)}=d \frac{1}{u(t)}+\omega \wedge \frac{1}{u(t)}=-\frac{d u(t)}{u(t)^{2}}+\frac{d u(t)}{u(t)^{2}}=0 .
$$

Since $1 / u(t)$ is not single valued on $T_{x}$ under $\alpha \in(\mathbb{C}-\mathbb{Z})^{m+3}$, its global solution is only 0 . Thus the spaces vanish.

Let us show $H^{2}\left(\mathcal{E}^{\bullet}, \nabla_{\omega}\right)=0$. For any $\eta \in \mathcal{E}^{2}$, we find $\psi \in \mathcal{E}^{1}$ such that $\nabla_{\omega} \psi=\eta$. Note that $\eta$ is $(1,1)$-form and $\bar{\partial} \eta=0$. By the $\bar{\partial}$-Poincaré lemma, there exists a $(1,0)$-form $\psi$ such that $\bar{\partial} \psi=\eta$. It takes the form $\psi=g(t) d t$ and satisfies

$$
\nabla_{\omega} \psi=(\partial+\bar{\partial}) \cdot(g(t) d t)+\omega \wedge(g(t) d t)=\bar{\partial}(g(t) d t)=\eta .
$$

Thus any element of $\mathcal{E}^{2}$ is a $\nabla_{\omega}$-image of $\mathcal{E}^{1}$, and $H^{2}\left(\mathcal{E}^{\bullet}, \nabla_{\omega}\right)=0$. Hence we have

$$
\operatorname{dim} H^{1}\left(\mathcal{E}^{\bullet}, \nabla_{\omega}\right)=-\chi\left(T_{x}\right)=m+1 .
$$

To obtain $H^{2}\left(\mathcal{E}_{c}^{\bullet}, \nabla_{\omega}\right)=0$, we show the natural map $H^{1}\left(\mathcal{E}_{c}^{\bullet}, \nabla_{\omega}\right) \rightarrow H^{1}\left(\mathcal{E}^{\bullet}, \nabla_{\omega}\right)$ is surjective. For any $\psi \in \mathcal{E}^{1}$ satisfying $\nabla_{\omega} \psi=0$, we find $\psi^{\prime} \in \mathcal{E}_{c}^{1}$ such that $\psi-\psi^{\prime} \in \nabla_{\omega}\left(\mathcal{E}^{0}\right)$. Set

$$
f_{j}(t)=\frac{1}{\left(e^{2 \pi \sqrt{-1}} \alpha_{j}-1\right) u(t)} \int_{C(t)} u(t) \psi,
$$

where $C(t)$ is a loop with terminal $t$ turning once around $x_{j}$ positively. It is independent of the choice of loops by $\nabla_{\omega} \psi=0$ and Twisted Stocks' theorem. Thus $f_{j}$ is single valued around $x_{j}$ and satisfies

$$
\begin{aligned}
& \nabla_{\omega}\left(f_{j}\right)=\frac{-d u(t)}{\left(e^{2 \pi \sqrt{-1}} \alpha_{j}-1\right) u(t)^{2}} \int_{C(t)} u(t) \psi+\frac{e^{2 \pi \sqrt{-1}} \alpha_{j} u(t) \psi-u(t) \psi}{\left(e^{2 \pi \sqrt{-1}} \alpha_{j}-1\right) u(t)} \\
& +\frac{d u(t)}{u(t)} \wedge \frac{1}{\left(e^{2 \pi \sqrt{-1}} \alpha_{j}-1\right) u(t)} \int_{C(t)} u(t) \psi \\
& =\psi \text {. }
\end{aligned}
$$


Let $h_{j}$ be a smooth function on $\mathbb{P}^{1}$ satisfying

$$
h_{j}(t)=\left\{\begin{array}{lll}
1 & \text { if } & t \in U_{j} \\
0 & \text { if } & t \in V_{j}^{c}
\end{array}\right.
$$

where $U_{j}$ and $V_{j}$ are neighborhoods of $x_{j}$ satisfying $U_{j} \subset V_{j}$. Though $f_{j}$ are defined only around $x_{j}$, the function $\sum_{j=0}^{m+2} h_{j}(t) f_{j}(t)$ can be regarded as a function on $T_{x}$.

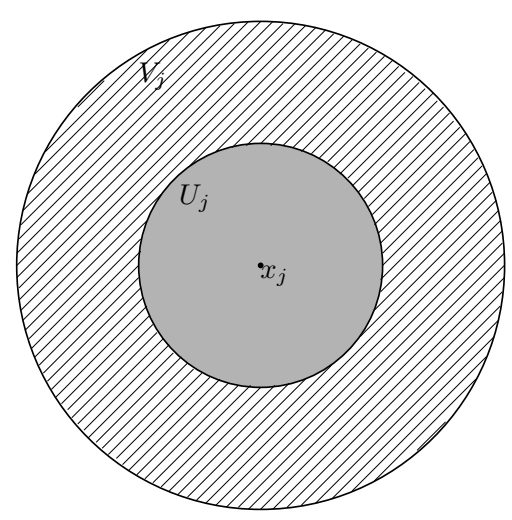

Since $f_{j}$ satisfies $\nabla_{\omega} f_{j}=\psi$ around $x_{j}$, and $h_{j} f_{j}$ identically vanishes around $x_{k}(j \neq k)$, we have

$$
\psi^{\prime}=\psi-\nabla_{\omega}\left(\sum_{j=0}^{m+2} h_{j}(t) f_{j}(t)\right) \in \mathcal{E}_{c}^{1}
$$

which shows that the natural map (13) is surjective.

Let us show $H^{2}\left(\mathcal{E}_{c}^{\bullet}, \nabla_{\omega}\right)=0$. For any $\eta \in \mathcal{E}_{c}^{2}$, there exists a $(1,0)$-form $\psi$ such that $\nabla_{\omega} \psi=\eta$. We have seen the existence of $\psi$ as an element of $\mathcal{E}_{1}, \psi$ may not belong to $\mathcal{E}_{c}^{1}$. However $\nabla_{\omega} \psi(=\eta)$ vanishes identically around $x_{j}(j=0, \ldots, m+2)$, there exists a smooth function $f_{j}$ around $x_{j}$ such that $\nabla_{\omega} f_{j}=\psi$ as in (14). Though $f_{j}$ is defined only around $x_{j}, h_{j}(t) f_{j}(t)$ can be regarded as defined on $T_{x}$. Thus we have

$$
\psi-\nabla_{\omega}\left(\sum_{j=0}^{m+2} h_{j}(t) f_{j}(t)\right) \in \mathcal{E}_{c}^{1}, \quad \nabla_{\omega}\left(\psi-\nabla_{\omega}\left(\sum_{j=0}^{m+2} h_{j}(t) f_{j}(t)\right)\right)=\eta
$$

which mean that $H^{2}\left(\mathcal{E}_{c}^{\bullet}, \nabla_{\omega}\right)=0$. Hence we have

$$
\operatorname{dim} H^{1}\left(\mathcal{E}_{c}^{\bullet}, \nabla_{\omega}\right)=-\chi\left(T_{x}\right)=m+1 .
$$

We have seen that the natural map $H^{1}\left(\mathcal{E}_{c}^{\bullet}, \nabla_{\omega}\right) \rightarrow H^{1}\left(\mathcal{E}^{\bullet}, \nabla_{\omega}\right)$ is surjective. Because of $\operatorname{dim} H^{1}\left(\mathcal{E}^{\bullet}, \nabla_{\omega}\right)=\operatorname{dim} H^{1}\left(\mathcal{E}_{c}^{\bullet}, \nabla_{\omega}\right)$, this map is isomorphic.

Definition 5. We define

$$
\iota_{\omega}: H^{1}\left(\mathcal{E}^{\bullet}, \nabla_{\omega}\right) \rightarrow H^{1}\left(\mathcal{E}_{c}^{\bullet}, \nabla_{\omega}\right)
$$

by the inverse of the natural map $H^{1}\left(\mathcal{E}_{c}^{\bullet}, \nabla_{\omega}\right) \rightarrow H^{1}\left(\mathcal{E}^{\bullet}, \nabla_{\omega}\right)$. 
Here we mention about the natural map $H^{1}\left(\mathcal{E}_{c}^{\bullet}, \nabla_{\omega}\right) \rightarrow H^{1}\left(\mathcal{E}^{\bullet}, \nabla_{\omega}\right)$ in case of $\alpha \notin \mathbb{Z}^{m+3}$ and $\alpha_{j} \in \mathbb{Z}$. We may assume $\alpha_{m+2} \notin \mathbb{Z}$ otherwise use a projective transformation sending $x_{k}$ with $\alpha_{k} \notin \mathbb{Z}$ to $\infty$. In this case, $f_{j}$ in (14) cannot be defined. Instead of the circle $C(t)$ in (14), we take a path $C_{\infty}(t)$ starting from $t$, approaching to $x_{m+2}=\infty$ turning this point once around positively, and tracing back to $t$. Then the function

$$
g_{j}(t)=\frac{1}{\left(e^{2 \pi \sqrt{-1}} \alpha_{m+2}-1\right) u(t)} \int_{C_{\infty}(t)} u(t) \psi
$$

is single valued on a small neighborhood of $t$, and satisfies $\nabla_{\omega} g_{j}(t)=\psi$. However, if $u(t) \psi$ includes the term $\frac{c d t}{t-x_{j}}$ then $c \log \left(t-x_{j}\right)$ appears and $g_{j}(t)$ cannot be single valued on $U_{j}$. Thus the natural map $H^{1}\left(\mathcal{E}_{c}^{\bullet}, \nabla_{\omega}\right) \rightarrow H^{1}\left(\mathcal{E}^{\bullet}, \nabla_{\omega}\right)$ is not surjective in this case.

In the proof of $H^{2}\left(\mathcal{E}_{c}^{\bullet}, \nabla_{\omega}\right)=0$, we can kill this term $\frac{c d t}{t-x_{j}}$ by adding a 1-form $\frac{-c d t}{\left(t-x_{j}\right)^{\alpha_{j}+1}}$ to $\psi$. Since $\nabla_{\omega}\left(\frac{-d t}{\left(t-x_{j}\right)^{\alpha_{j}+1}}\right)=0$, the property $\nabla_{\omega} \psi=\eta$ is kept by this addition. Hence we obtain $H^{2}\left(\mathcal{E}_{c}^{\bullet}, \nabla_{\omega}\right)=0$ in this case. Thus we have the following proposition.

Proposition 4. If $\alpha \notin \mathbb{Z}^{m+3}$ then

$$
\begin{aligned}
H^{0}\left(\mathcal{E}^{\bullet}, \nabla_{\omega}\right)= & 0, \quad H^{2}\left(\mathcal{E}^{\bullet}, \nabla_{\omega}\right)=0, \quad H^{0}\left(\mathcal{E}_{c}^{\bullet}, \nabla_{\omega}\right)=0, \quad H^{2}\left(\mathcal{E}_{c}^{\bullet}, \nabla_{\omega}\right)=0, \\
\operatorname{dim} H^{1}\left(\mathcal{E}^{\bullet}, \nabla_{\omega}\right) & =\operatorname{dim} H^{1}\left(\mathcal{E}_{c}^{\bullet}, \nabla_{\omega}\right)=m+1 .
\end{aligned}
$$

In the above observation, we see that if $\alpha \notin \mathbb{Z}^{m+3}$ and $\alpha_{j} \in \mathbb{Z}$ then the natural map $H^{1}\left(\mathcal{E}_{c}^{\bullet}, \nabla_{\omega}\right) \rightarrow H^{1}\left(\mathcal{E}^{\bullet}, \nabla_{\omega}\right)$ is not surjective though $\operatorname{dim} H^{1}\left(\mathcal{E}^{\bullet}, \nabla_{\omega}\right)=\operatorname{dim} H^{1}\left(\mathcal{E}_{c}^{\bullet}, \nabla_{\omega}\right)$. We given an element of the kernel of the natural map in this case. Note that

$$
\frac{d h_{j}(t)}{u(t)} \in \mathcal{E}_{c}^{1}, \quad \frac{h_{j}(t)}{u(t)} \in \mathcal{E}^{0}-\mathcal{E}_{c}^{0}
$$

where $h_{j}(t)$ is given in (15). Since

$$
\begin{aligned}
\nabla_{\omega}\left(\frac{d h_{j}(t)}{u(t)}\right) & =\frac{d \circ d\left(h_{j}(t)\right)}{u(t)}-d h_{j}(t) \wedge d\left(\frac{1}{u(t)}\right)+\omega \wedge \frac{d h_{j}(t)}{u(t)} \\
& =-d h_{j}(t) \wedge \nabla_{\omega}\left(\frac{1}{u(t)}\right)=0 \\
\nabla_{\omega}\left(\frac{h_{j}(t)}{u(t)}\right) & =\frac{d\left(h_{j}(t)\right)}{u(t)}+h_{j}(t) \cdot d\left(\frac{1}{u(t)}\right)+\omega \wedge \frac{h_{j}(t)}{u(t)} \\
& =\frac{d h_{j}(t)}{u(t)}+h_{j}(t) \cdot \nabla_{\omega}\left(\frac{1}{u(t)}\right)=\frac{d h_{j}(t)}{u(t)}
\end{aligned}
$$

$\frac{d h_{j}(t)}{u(t)}$ is $\nabla_{\omega}$-closed and belongs to $\nabla_{\omega}\left(\mathcal{E}^{0}\right)$. Thus $\frac{d h_{j}(t)}{u(t)}$ is zero as elements of $H^{1}\left(\mathcal{E}^{\bullet}, \nabla_{\omega}\right)$. Recall that $\operatorname{ker}\left(\nabla_{\omega}: \mathcal{E}^{0} \rightarrow \mathcal{E}^{1}\right)$ is spanned by $1 / u(t)$, which is not single valued on $T_{x}$ under $\alpha \notin \mathbb{Z}^{m+3}$. Thus the global solution to $\nabla_{\omega}(f)=\frac{d h_{j}(t)}{u(t)}$ on $T_{x}$ is unique. Since $\frac{h_{j}(t)}{u(t)} \notin \mathcal{E}_{c}^{0}, \frac{d h_{j}(t)}{u(t)}$ is different from 0 as elements of $H^{1}\left(\mathcal{E}_{c}^{\bullet}, \nabla_{\omega}\right)$. Hence this is a non-zero element in the kernel of the natural map. 
Remark 3. Since there exists $\psi \in \mathcal{E}^{1}$ such that $\nabla_{\omega} \psi=\eta$ for any $\eta \in \mathcal{E}^{2}$, and $1 / u(t)$ does not have a compact support,

$$
H^{2}\left(\mathcal{E}^{\bullet}, \nabla_{\omega}\right)=0, \quad H^{0}\left(\mathcal{E}_{c}^{\bullet}, \nabla_{\omega}\right)=0
$$

without any conditions on $\alpha$.

\section{Intersection form between twisted cohomology groups}

By using $\nabla_{-\omega}=d-\omega \wedge$ instead of $\nabla_{\omega}$, we have twisted cohomology groups $H^{k}\left(\mathcal{E}^{\bullet}, \nabla_{-\omega}\right)$ $(k=0,1,2)$, which satisfy

$$
H^{0}\left(\mathcal{E}^{\bullet}, \nabla_{-\omega}\right)=0, \quad H^{2}\left(\mathcal{E}^{\bullet}, \nabla_{-\omega}\right)=0, \quad \operatorname{dim} H^{1}\left(\mathcal{E}^{\bullet}, \nabla_{-\omega}\right)=m+1 .
$$

Definition 6 (Intersection number of $\nabla_{ \pm \omega}$-closed 1-forms). The intersection number of $\varphi \in \operatorname{ker}\left(\nabla_{\omega}\right.$ : $\left.\mathcal{E}_{c}^{1} \rightarrow \mathcal{E}_{c}^{2}\right)$ and $\psi \in \operatorname{ker}\left(\nabla_{-\omega}: \mathcal{E}^{1} \rightarrow \mathcal{E}^{2}\right)$ is defined by

$$
\langle\varphi, \psi\rangle=\iint_{T_{x}} \varphi \wedge \psi
$$

It induces a bi-linear form between the space of $\nabla_{\omega}$-closed 1 -forms and that of $\nabla_{-\omega}$-closed 1 -forms.

Since $\varphi \in \mathcal{E}_{c}^{1}$ has a compact support, the support of $\varphi \wedge \psi$ is also compact, and the integral in (16) converges.

Theorem 4. The intersection form between $\operatorname{ker}\left(\nabla_{\omega}: \mathcal{E}_{c}^{1} \rightarrow \mathcal{E}_{c}^{2}\right)$ and $\operatorname{ker}\left(\nabla_{-\omega}: \mathcal{E}^{1} \rightarrow \mathcal{E}^{2}\right)$ descends to that between $H^{1}\left(\mathcal{E}_{c}^{\bullet}, \nabla_{\omega}\right)$ and $H^{1}\left(\mathcal{E}^{\bullet}, \nabla_{-\omega}\right)$. The isomorphism $\imath_{\omega}: H^{1}\left(\mathcal{E}^{\bullet}, \nabla_{\omega}\right) \rightarrow H^{1}\left(\mathcal{E}_{c}^{\bullet}, \nabla_{\omega}\right)$ given in Definition 5 induces the intersection form between $H^{1}\left(\mathcal{E}^{\bullet}, \nabla_{\omega}\right)$ and $H^{1}\left(\mathcal{E}^{\bullet}, \nabla_{-\omega}\right)$ by

$$
\langle\varphi, \psi\rangle=\iint_{T_{x}} l_{\omega}(\varphi) \wedge \psi
$$

Proof. For a $\nabla_{-\omega}$-closed 1-form $\psi \in \mathcal{E}^{1}, f \in \mathcal{E}_{c}^{0}$, we have

$$
\begin{aligned}
& \iint_{T_{x}}\left(\nabla_{\omega} f\right) \wedge \psi=\iint_{T_{x}}(d f+\omega \wedge f) \wedge \psi=\iint_{T_{x}}(d f) \wedge \psi+f \cdot \omega \wedge \psi \\
= & \iint_{T_{x}}(d(f \cdot \psi)-f \cdot d \psi)+f \cdot \omega \wedge \psi=\iint_{T_{x}} d(f \cdot \psi)-f \cdot \nabla_{-\omega} \psi \\
= & \int_{\partial T_{x}} f \cdot \psi-\iint_{T_{x}} f \cdot 0=0,
\end{aligned}
$$

since $f \cdot \psi \in \mathcal{E}_{c}^{1}$. Similarly, we can show $\iint_{T_{x}} \varphi \wedge\left(\nabla_{-\omega} g\right)=0$ for a $\nabla_{\omega}$-closed 1-form $\varphi \in \mathcal{E}_{c}^{1}$, $g \in \mathcal{E}^{0}$. Thus $\langle$,$\rangle descends to the intersection form between H^{1}\left(\mathcal{E}_{c}^{\bullet}, \nabla_{\omega}\right)$ and $H^{1}\left(\mathcal{E}^{\bullet}, \nabla_{-\omega}\right)$.

Though $\iint_{T_{x}} \varphi \wedge \psi$ is not well defined for a $\nabla_{\omega^{-}}$closed 1-form $\varphi \in \mathcal{E}^{1}$ and a $\nabla_{-\omega^{-}}$-closed 1-form $\psi \in \mathcal{E}^{1},\langle$,$\rangle is a well-defined form between H^{1}\left(\mathcal{E}_{c}^{\bullet}, \nabla_{\omega}\right)$ and $H^{1}\left(\mathcal{E}^{\bullet}, \nabla_{-\omega}\right)$ and $\iota_{\omega}$ is isomorphic. Thus $\langle$,$\rangle can be extended to the intersection form between H^{1}\left(\mathcal{E}^{\bullet}, \nabla_{\omega}\right)$ and $H^{1}\left(\mathcal{E}^{\bullet}, \nabla_{-\omega}\right)$. 
Theorem 5 ([CM, Theorem 1],[M1, §4]). The intersection form is non-degenerate. For

$$
\varphi_{j}=d \log \frac{t-x_{j}}{t-x_{j+1}}, \quad \psi_{k}=d \log \frac{t-x_{k}}{t-x_{k+1}}
$$

we have

$$
\left\langle\varphi_{j}, \psi_{k}\right\rangle=2 \pi \sqrt{-1}\left\{\begin{array}{cl}
\frac{-1}{\alpha_{j}} & \text { if } k=j-1, \\
\frac{\alpha_{j}+\alpha_{j+1}}{\alpha_{j} \alpha_{j+1}} & \text { if } k=j, \\
\frac{-1}{\alpha_{j+1}} & \text { if } k=j+1, \\
0 & \text { otherwise. }
\end{array}\right.
$$

Proof. We consider $\iota_{\omega}: H^{1}\left(\mathcal{E}^{\bullet}, \nabla_{\omega}\right) \rightarrow H^{1}\left(\mathcal{E}_{c}^{\bullet}, \nabla_{\omega}\right)$. Note that $\varphi_{j}$ is a rational 1 -form admitting poles only on $x_{j}, x_{j+1}$ with residues $1,-1$ on $\mathbb{P}^{1}$. Recall that we have expressed a solution to $\nabla_{\omega}(f)=\psi$ by the integral in (14). In this time, we express a solution $f_{\ell}$ to $\nabla_{\omega} f_{\ell}=\varphi_{j}$ in $V_{\ell}$ as a power series.

Put $f_{\ell}(t)=\sum_{n=0}^{\infty} a_{n}\left(t-x_{\ell}\right)^{n}$ with unknowns $a_{n}$ 's in $V_{\ell}$. Then we can determine uniquely $a_{n}$ by comparing coefficients of the both sides of $\nabla_{\omega} f_{\ell}=\varphi_{j}$. Since the Laurent expansion of $\omega$ around $t=x_{\ell}$ is

$$
\omega=\left[\frac{\alpha_{\ell}}{t-x_{\ell}}+b_{0}+b_{1}\left(t-x_{\ell}\right)+\cdots\right] d t
$$

the starting term $a_{0}$ of $f_{\ell}(t)$ is

$$
a_{0}=\left\{\begin{aligned}
0 & \text { if } \quad \ell \neq j, j+1, \\
\frac{1}{\alpha_{\ell}} & \text { if } \quad \ell=j, \\
\frac{-1}{\alpha_{\ell+1}} & \text { if } \quad \ell=j+1 .
\end{aligned}\right.
$$

By the existence of a solution, each series has a positive radius of convergence.

We can regard

$$
f=\sum_{\ell=0}^{m+2} h_{\ell} f_{\ell}
$$

as a function on $T_{x}$, where $h_{\ell}$ is given in (15). Note that $\varphi_{j}-\nabla_{\omega} f$ vanishes identically on $U_{\ell}$, and $\varphi_{j}-\nabla_{\omega} f \in \mathcal{E}_{c}^{1}$. Hence $\imath_{\omega}\left(\varphi_{j}\right)$ is represented by $\varphi_{j}-\nabla_{\omega} f$, and $\varphi_{j}-\nabla_{\omega} f=\varphi_{j}$ on the complement 
of $\cup_{\ell=0}^{m+2} V_{\ell}$. Thus we have

$$
\begin{aligned}
\left\langle\varphi_{j}, \psi_{k}\right\rangle & =\iint_{T_{x}}\left(\varphi_{j}-\nabla_{\omega} f\right) \wedge \psi_{k}=\sum_{\ell=0}^{m+2} \iint_{V_{\ell}-U_{\ell}}\left(\varphi_{j}-\nabla_{\omega} h_{\ell} f_{\ell}\right) \wedge \psi_{k} \\
& =-\sum_{\ell=0}^{m+2} \iint_{V_{\ell}-U_{\ell}}\left(\nabla_{\omega} h_{\ell} f_{\ell}\right) \wedge \psi_{k}=-\sum_{\ell=0}^{m+2} \iint_{V_{\ell}-U_{\ell}}\left(f_{\ell} d h_{\ell}+h_{\ell}\left(\nabla_{\omega} f_{\ell}\right)\right) \wedge \psi_{k} \\
& =-\sum_{\ell=0}^{m+2} \iint_{V_{\ell}-U_{\ell}} f_{\ell} d h_{\ell} \wedge \psi_{k}=-\sum_{\ell=0}^{m+2} \int_{\partial\left(V_{\ell}-U_{\ell}\right)} h_{\ell} f_{\ell} \psi_{k}=\sum_{\ell=0}^{m+2} \int_{\partial U_{\ell}} f_{\ell} \psi_{k} \\
& =2 \pi \sqrt{-1} \sum_{\ell=0}^{m+2} \operatorname{Res}_{t=x_{\ell}}\left(f_{\ell} \psi_{k}\right) .
\end{aligned}
$$

Here we use Stokes' Theorem and Residue Theorem. Note that $h_{\ell}$ vanishes identically on $\partial V_{\ell}$, and becomes identically 1 on $\partial U_{\ell}$. Residue calculuses together with (17) yield the expression of $\left\langle\varphi_{j}, \psi_{k}\right\rangle$ in this proposition.

We have the intersection matrix

$$
H_{c}=\left(\left\langle\varphi_{j}, \psi_{k}\right\rangle\right)_{0 \leq j, k \leq m}=2 \pi \sqrt{-1}\left(\begin{array}{ccccc}
\frac{\alpha_{0}+\alpha_{1}}{\alpha_{0} \alpha_{1}} & \frac{-1}{\alpha_{1}} & 0 & \cdots & 0 \\
\frac{-1}{\alpha_{1}} & \frac{\alpha_{1}+\alpha_{2}}{\alpha_{1} \alpha_{2}} & \frac{-1}{\alpha_{2}} & \ddots & \\
0 & \frac{-1}{\alpha_{2}} & \frac{\alpha_{2}+\alpha_{3}}{\alpha_{2} \alpha_{3}} & \ddots & \vdots \\
\vdots & \ddots & \ddots & \ddots & \\
0 & & \cdots & & \frac{\alpha_{m}+\alpha_{m+1}}{\alpha_{m} \alpha_{m+1}}
\end{array}\right)
$$

with $\operatorname{det}\left(H_{c}\right)=\frac{-(2 \pi \sqrt{-1})^{m+1} \alpha_{m+2}}{\alpha_{0} \alpha_{1} \cdots \alpha_{m+1}} \neq 0$. Therefore the intersection form is non-degenerate.

\section{Twisted period relation}

There is a natural pairing between the twisted cohomology group $H^{1}\left(\mathcal{E}^{\bullet}, \nabla_{\omega}\right)$ and the twisted homology group $H_{1}\left(C_{\bullet}^{u}, \partial_{\omega}\right)$ defined by

$$
\langle\varphi, \gamma\rangle=\sum_{i} a_{i} \int_{I_{i}} u_{I_{i}}(t) \varphi
$$

where $\varphi \in H^{1}\left(\mathcal{E}^{\bullet}, \nabla_{\omega}\right)$ and $\gamma=\sum_{i} a_{i} I_{i} \otimes u_{I_{i}}(t) \in H_{1}\left(C_{\bullet}^{u}, \partial_{\omega}\right)$. It is known that this pairing is perfect, and yields solutions to Lauricella's hypergeometric system $\mathcal{F}_{D}(a, b, c)$. Similarly, we have a natural perfect pairing

$$
\langle\psi, \delta\rangle=\sum_{j} b_{j} \int_{J_{j}} u_{J_{j}}^{-1}(t) \psi,
$$

between $\psi \in H^{1}\left(\mathcal{E}^{\bullet}, \nabla_{-\omega}\right)$ and $\delta=\sum_{j} b_{j} J_{j} \otimes u_{J_{j}}^{-1}(t) \in H_{1}\left(C_{\bullet}^{u^{-1}}, \partial_{-\omega}\right)$.

We can regard $H^{1}\left(\mathcal{E}^{\bullet}, \nabla_{\omega}\right)$ and $H_{1}\left(C_{\bullet}^{u^{-1}}, \partial_{-\omega}\right)$ as dual spaces of $H^{1}\left(\mathcal{E}^{\bullet}, \nabla_{-\omega}\right)$ with respect to the intersection form between twisted cohomology groups, and to (19), respectively. Thus we have an isomorphism iso $_{c}: H^{1}\left(\mathcal{E}^{\bullet}, \nabla_{\omega}\right) \rightarrow H_{1}\left(C_{\bullet}^{u^{-1}}, \partial_{-\omega}\right)$ such that

$$
\langle\varphi, \psi\rangle=\left\langle\psi, i \operatorname{soc}_{c}(\varphi)\right\rangle
$$


for any $\psi \in H^{1}\left(\mathcal{E}^{\bullet}, \nabla_{-\omega}\right)$. On the other hand, it is also possible to regard them as dual spaces of $H_{1}\left(C_{\bullet}^{u}, \partial_{\omega}\right)$ with respect to (18), and to the intersection form between twisted homology groups, respectively. There is an isomorphism $i s o_{h}: H^{1}\left(\mathcal{E}^{\bullet}, \nabla_{\omega}\right) \rightarrow H_{1}\left(C_{\bullet}^{u^{-1}}, \partial_{-\omega}\right)$ such that

$$
\langle\varphi, \gamma\rangle=\left\langle\gamma, \operatorname{iso}_{h}(\varphi)\right\rangle
$$

for any $\gamma \in H_{1}\left(C_{\bullet}^{u}, \partial_{\omega}\right)$. See the diagram below.

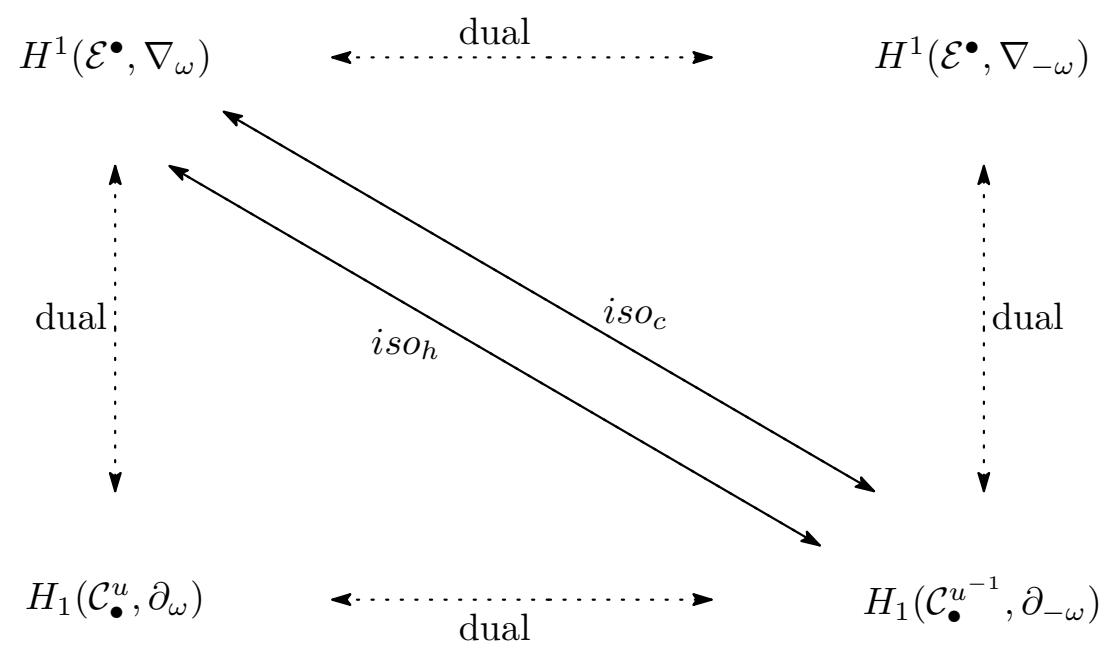

As shown in [KY1, §1] and [CM, §3], the following theorem holds.

Theorem 6 (Compatibility of intersection forms). The isomorphisms iso $o_{c}$ and iso $\mathrm{s}_{h}$ coincide.

Remark 4. Definition 3 does not directly yield a bilinear form between the spaces of twisted 1cycles. Note that there is a case that simplexes $I_{i}$ and $J_{j}$ do not intersect transversally, where $I_{i}$ and $J_{j}$ are 1-simplexes of twisted 1-cycles $\gamma=\sum_{i} a_{i} I_{i} \otimes u(t)$ and $\delta=\sum_{j} b_{j} J_{j} \otimes u^{-1}(t)$, respectively. By using $i s o_{c}$, we can define the intersection form between twisted homology groups $H_{1}\left(C_{\bullet}^{u}, \partial_{\omega}\right)$ and $H_{1}\left(C_{\bullet}^{u^{-1}}, \partial_{-\omega}\right)$ by

$$
\langle\gamma, \delta\rangle=\left\langle i s o_{c}^{-1}(\delta), \gamma\right\rangle=\sum_{i} a_{i} \int_{I_{i}} u(t) i s o_{c}^{-1}(\gamma) .
$$

Though there is no ambiguity in this definition, we cannot directly evaluate $\langle\gamma, \delta\rangle$ by this definition. We can show that (20) is equal to the value given in Definition 3, refer to [M3, §7].

Corollary 1 ([CM, Theorem 2]). For bases ${ }^{t}\left(\varphi_{0}, \ldots, \varphi_{m}\right),\left(\psi_{0}, \ldots, \psi_{m}\right),\left(\gamma_{0}, \ldots, \gamma_{m}\right),{ }^{t}\left(\delta_{0}, \ldots, \delta_{m}\right)$ of $H^{1}\left(\mathcal{E}^{\bullet}, \nabla_{\omega}\right), H^{1}\left(\mathcal{E}^{\bullet}, \nabla_{-\omega}\right), H_{1}\left(C_{\bullet}^{u}, \partial_{\omega}\right), H_{1}\left(C_{\bullet}^{u^{-1}}, \partial_{-\omega}\right)$, construct four $(m+1) \times(m+1)$ matrices

$$
\Pi_{\omega}=\left(\left\langle\varphi_{j}, \gamma_{k}\right\rangle\right), \quad \Pi_{-\omega}=\left(\left\langle\psi_{j}, \delta_{k}\right\rangle\right), \quad H_{c}=\left(\left\langle\varphi_{j}, \psi_{k}\right\rangle\right), \quad H_{h}=\left(\left\langle\gamma_{j}, \delta_{k}\right\rangle\right) .
$$

Then they satisfy a twisted period relation

$$
\Pi_{\omega}{ }^{t} H_{h}^{-1}{ }^{t} \Pi_{-\omega}=H_{c}, \text { i.e. } \quad{ }^{t} \Pi_{-\omega} H_{c}^{-1} \Pi_{\omega}={ }^{t} H_{h} .
$$

Proof. By Theorem 6, we show this corollary. Let $M$ be the representation matrix of $i s o_{c}\left(=i s o_{h}\right)$ with respect to bases ${ }^{t}\left(\varphi_{0}, \ldots, \varphi_{m}\right)$ and ${ }^{t}\left(\delta_{0}, \ldots, \delta_{m}\right)$, i.e.,

$$
{ }^{t}\left(\operatorname{iso}_{h}\left(\varphi_{0}\right), \ldots, i \operatorname{son}_{h}\left(\varphi_{m}\right)\right)=M^{t}\left(\delta_{0}, \ldots, \delta_{m}\right) .
$$


We have

$$
\begin{aligned}
H_{c} & =\left(\left\langle\varphi_{j}, \psi_{k}\right\rangle\right)_{j, k}={ }^{t}\left(\varphi_{0}, \ldots, \varphi_{m}\right) \cdot\left(\psi_{0}, \ldots, \psi_{m}\right)={ }^{t}\left(\operatorname{iso}_{c}\left(\varphi_{0}\right), \ldots, i \operatorname{so}_{c}\left(\varphi_{m}\right)\right) \cdot\left(\psi_{0}, \ldots, \psi_{m}\right) \\
& =M^{t}\left(\delta_{0}, \ldots, \delta_{m}\right) \cdot\left(\psi_{0}, \ldots, \psi_{m}\right)=M^{t} \Pi_{-\omega},
\end{aligned}
$$

where $\cdot$ denotes the pairing with matrix arrangement. Similarly, we have

$$
\begin{aligned}
\Pi_{\omega} & =\left(\left\langle\varphi_{j}, \gamma_{k}\right\rangle\right)_{j, k}={ }^{t}\left(\varphi_{0}, \ldots, \varphi_{m}\right) \cdot\left(\gamma_{0}, \ldots, \gamma_{m}\right)={ }^{t}\left(i s o_{h}\left(\varphi_{0}\right), \ldots, i s o_{h}\left(\varphi_{m}\right)\right) \cdot\left(\gamma_{0}, \ldots, \gamma_{m}\right) \\
& =M^{t}\left(\delta_{0}, \ldots, \delta_{m}\right) \cdot\left(\gamma_{0}, \ldots, \gamma_{m}\right)=M^{t} H_{h} .
\end{aligned}
$$

By eliminating $M$ from two identities $H_{c}=M^{t} \Pi_{-\omega}$ and $\Pi_{\omega}=M^{t} H_{h}$, we have $H_{c}=$ $\Pi_{\omega}{ }^{t} H_{h}^{-1}{ }^{t} \Pi_{-\omega}$.

We explain several examples in [CM, §4]. In case of $m=0$, the twisted (co)homology groups in Corollary 1 are one dimensional, where $T_{x}=\mathbb{P}^{1}-\{0,1, \infty\}$ and $u(t)=t^{p}(1-t)^{q}$, $1 / u(t)=t^{-p}(1-t)^{-q}$. Take bases of $H^{1}\left(\mathcal{E}^{\bullet}, \nabla_{\omega}\right), H^{1}\left(\mathcal{E}^{\bullet}, \nabla_{-\omega}\right), H_{1}\left(C_{\bullet}^{u}, \partial_{\omega}\right), H_{1}\left(C_{\bullet}^{u^{-1}}, \partial_{-\omega}\right)$ as $\varphi_{0}=\frac{d t}{t(1-t)}, \psi_{0}=\frac{d t}{t(1-t)}, \gamma_{0}$ given in (11), $\delta_{0}$ replaced $p, q$ into $-p,-q$ for $\gamma_{0}$, respectively. Note that

$$
\left\langle\varphi_{0}, \gamma_{0}\right\rangle=B(p, q), \quad\left\langle\psi_{0}, \delta_{0}\right\rangle=B(-p,-q)
$$

by (18), (19) and Proposition 1, and that they are well defined under $p, q \notin \mathbb{Z}$. By Theorems 3 and 5 , we have

$$
\left\langle\varphi_{0}, \psi_{0}\right\rangle=\frac{2 \pi \sqrt{-1}(p+q)}{p q}, \quad\left\langle\gamma_{0}, \delta_{0}\right\rangle=\frac{1-e^{2 \pi \sqrt{-1}(p+q)}}{\left(1-e^{2 \pi \sqrt{-1}} p\right)\left(1-e^{2 \pi \sqrt{-1} q}\right)} .
$$

In this case, the twisted period relation (21) is equivalent to $\left\langle\varphi_{0}, \gamma_{0}\right\rangle\left\langle\psi_{0}, \delta_{0}\right\rangle=\left\langle\varphi_{0}, \psi_{0}\right\rangle\left\langle\gamma_{0}, \delta_{0}\right\rangle$, which yields the inversion formula (2) for the Beta function.

In case of $m=1$, we derive identities for hypergeometric functions from the twisted period relation. Recall our setting

$$
\begin{gathered}
x_{0}=0, \quad x=x_{1}\left(0<x_{1}<1\right), \quad x_{2}=1, \quad x_{3}=\infty, \\
\alpha_{0}=b-c, \quad \alpha_{1}=-b, \quad \alpha_{2}=c-a, \quad \alpha_{3}=a, \\
u(t)=t^{b-c}(t-x)^{-b}(t-1)^{c-a}, \quad \omega=d \log u(t) .
\end{gathered}
$$

To simplify the twisted period relation, we assume $\alpha_{2}+\alpha_{3}=c \notin \mathbb{Z}$. We select $\nabla_{\omega}$-closed forms

$$
\varphi_{2}=d \log \frac{t-x_{2}}{t-x_{3}}=\frac{d t}{t-1}, \quad \varphi_{0}=d \log \frac{t-x_{0}}{t-x_{1}}=\frac{-x d t}{t(t-x)}
$$

and they are $\nabla_{-\omega}$-closed. Let $\gamma_{2}, \gamma_{0}$ and $\delta_{2}, \delta_{0}$ be bases of $H_{1}\left(C_{\bullet}^{u}, \partial_{\omega}\right)$ and $H_{1}\left(C_{\bullet}^{u^{-1}}, \partial_{-\omega}\right)$. Here recall that the twisted cycles $\gamma_{j}$ and $\delta_{j}$ are given by the path $I_{j}$ from $x_{j}$ to $x_{j+1}$ and branches of $u(t)$ and $1 / u(t)$ on them, respectively. We have four matrices:

$$
\Pi_{\omega}=\left(\begin{array}{ll}
\left\langle\varphi_{2}, \gamma_{2}\right\rangle & \left\langle\varphi_{2}, \gamma_{0}\right\rangle \\
\left\langle\varphi_{0}, \gamma_{2}\right\rangle & \left\langle\varphi_{0}, \gamma_{0}\right\rangle
\end{array}\right), \quad \Pi_{-\omega}=\left(\begin{array}{cc}
\left\langle\varphi_{2}, \delta_{2}\right\rangle & \left\langle\varphi_{2}, \delta_{0}\right\rangle \\
\left\langle\varphi_{0}, \delta_{2}\right\rangle & \left\langle\varphi_{0}, \delta_{0}\right\rangle
\end{array}\right),
$$




$$
H_{c}=2 \pi \sqrt{-1}\left(\begin{array}{cc}
\frac{\alpha_{2}+\alpha_{3}}{\alpha_{2} \alpha_{3}} & 0 \\
0 & \frac{\alpha_{0}+\alpha_{1}}{\alpha_{0} \alpha_{1}}
\end{array}\right), \quad H_{h}=\left(\begin{array}{cc}
\frac{1-\theta_{2} \theta_{3}}{\left(1-\theta_{2}\right)\left(1-\theta_{3}\right)} & 0 \\
0 & \frac{1-\theta_{0} \theta_{1}}{\left(1-\theta_{0}\right)\left(1-\theta_{1}\right)}
\end{array}\right) .
$$

Any entry of $\Pi_{ \pm \omega}$ can be expressed by the hypergeometric function. For example,

$$
\begin{aligned}
& \left\langle\varphi_{2}, \gamma_{2}\right\rangle=B(a, c-a) F(a, b, c ; x) \\
& \left\langle\varphi_{2}, \gamma_{0}\right\rangle=-e^{\pi \sqrt{-1}(c-a-b)} x^{1-c} B(b-c+1,-b+1) F(b-c+1, a-c+1,2-c ; x) .
\end{aligned}
$$

Here note that the second is given by the variable change $t=x / s$. By the $(1,2)$-entry of the first identity of (21) in Corollary 1, we have

$$
F(a, b, c ; x) F(1-a, 1-b, 2-c ; x)=F(b-c+1, a-c+1,2-c ; x) F(c-a, c-b, c ; x) .
$$

By its $(1,1)$-entry, we have

$$
\begin{aligned}
& F(a, b, c ; x) F(-a,-b,-c ; x)-1 \\
= & \frac{a b(c-a)(c-b)}{c^{2}(c+1)(c-1)} x^{2} F(b-c+1, a-c+1,2-c ; x) F(c-b+1, c-a+1,2+c ; x) .
\end{aligned}
$$

In case of general $m$, the twisted period relation yields identities among some values of Lauricella's hypergeometric series $F_{D}$ with several parameters. Recall our setting

$$
\begin{gathered}
x_{0}=0, \quad x=\left(x_{1}, \ldots, x_{m}\right) \in \mathbb{D}_{m}, \quad x_{m+1}=1, \quad x_{m+2}=\infty, \\
\alpha_{0}=-c+\sum_{j=1}^{m} b_{j}, \quad \alpha_{j}=-b_{j}(1 \leq j \leq m), \quad \alpha_{m+1}=c-a, \quad \alpha_{m+2}=a, \\
u=u(t)=t^{b_{1}+\cdots+b_{m}-c}\left(t-x_{1}\right)^{-b_{1}} \cdots\left(t-x_{m}\right)^{-b_{m}}(t-1)^{c-a} . \\
\varphi_{0}=\frac{d t}{t-1}, \quad \varphi_{j}=\frac{d t}{t-x_{j}}, \\
\psi_{0}=\frac{d t}{t(t-1)}, \quad \psi_{j}=\frac{1}{x_{j}}\left(\frac{d t}{t-x_{j}}-\frac{d t}{t-x_{0}}\right)=\frac{d t}{t\left(t-x_{j}\right)},
\end{gathered}
$$

where $1 \leq j \leq m$ and the index start from 0 for bases of twisted (co)homology groups. We assume that $\alpha_{m+1}+\alpha_{m+2}=c \notin \mathbb{Z}$.

We set twisted cycles $\gamma_{0}$ and $\delta_{0}$ by the regularization of the open interval $(1, \infty)$ with respect to $u(t)$ and that to $1 / u(t)$, respectively. For any bases of $H_{1}\left(C_{\bullet}^{u}, \partial_{\omega}\right)$ and $H_{1}\left(C_{\bullet}^{u^{-1}}, \partial_{-\omega}\right)$ including $\gamma_{0}$ and $\delta_{0}$, the identity for the $(0,0)$-entry of ${ }^{t} \Pi_{-\omega} H_{c}^{-1} \Pi_{\omega}={ }^{t} H_{h}$ is

$$
\begin{aligned}
& \left(\int_{1}^{\infty} u^{-1} \psi_{0}, \ldots, \int_{1}^{\infty} u^{-1} \psi_{m}\right) H_{c}^{-1 t}\left(\int_{1}^{\infty} u \varphi_{0}, \ldots, \int_{1}^{\infty} u \varphi_{m}\right) \\
= & (0,0) \text {-entry of } H_{h}=\frac{1-e^{2 \pi \sqrt{-1} c}}{\left(1-e^{2 \pi \sqrt{-1}(c-a)}\right)\left(1-e^{2 \pi \sqrt{-1} a}\right)} .
\end{aligned}
$$

The intersection matrix $H_{c}$ is diagonal and

$$
H_{c}^{-1}=\frac{-1}{2 \pi \sqrt{-1}} \operatorname{diag}\left(a-c, b_{1} x_{1}, \ldots, b_{m} x_{m}\right) .
$$


The integrals are expressed as

$$
\begin{gathered}
\left(\begin{array}{c}
\int_{1}^{\infty} u \varphi_{0} \\
\int_{1}^{\infty} u \varphi_{1} \\
\vdots \\
\int_{1}^{\infty} u \varphi_{m}
\end{array}\right)=\left(\begin{array}{c}
B(a, c-a) F_{D}(a, b, c ; x) \\
B(a, c+1-a) F_{D}\left(a, b+e_{1}, c+1 ; x\right) \\
\vdots \\
B(a, c+1-a) F_{D}\left(a, b+e_{1}, c+1 ; x\right)
\end{array}\right), \\
\left(\begin{array}{c}
\int_{1}^{\infty} u^{-1} \psi_{0} \\
\int_{1}^{\infty} u^{-1} \psi_{1} \\
\vdots \\
\int_{1}^{\infty} u^{-1} \psi_{m}
\end{array}\right)=\left(\begin{array}{c}
B(1-a, a-c) F_{D}(1-a,-b, 1-c ; x) \\
B(1-a, a-c+1) F_{D}\left(1-a, e_{1}-b, 2-c ; x\right) \\
\vdots \\
B(1-a, a-c+1) F_{D}\left(1-a, e_{m}-b, 2-c ; x\right)
\end{array}\right),
\end{gathered}
$$

where $e_{j}$ is the $j$-th unit vector of size $m$. By using the inversion formula for the Beta function

$$
\begin{aligned}
B(a, c-a) B(1-a, a-c) & =B(a, c-a) \cdot\left(\frac{-a}{-c}\right) B(-a, a-c) \\
& =2 \pi \sqrt{-1} \frac{1}{c-a} \cdot \frac{1-e^{2 \pi \sqrt{-1} c}}{\left(1-e^{2 \pi \sqrt{-1}(c-a)}\right)\left(1-e^{2 \pi \sqrt{-1} a}\right)}, \\
B(a, c-a+1) B(1-a, a-c+1) & =\left(\frac{c-a}{c} \cdot B(a, c-a)\right) \cdot\left(\frac{-a(a-c)}{-c(1-c)} B(-a, a-c)\right) \\
& =2 \pi \sqrt{-1} \frac{a-c}{c(1-c)} \cdot \frac{1-e^{2 \pi \sqrt{-1} c}}{\left(1-e^{2 \pi \sqrt{-1}(c-a)}\right)\left(1-e^{2 \pi \sqrt{-1} a}\right)},
\end{aligned}
$$

we have an identity

$$
\begin{aligned}
& F_{D}(a, b, c ; x) F_{D}(1-a,-b, 1-c ; x)-1 \\
= & \frac{c-a}{c(c-1)} \sum_{j=1}^{m} b_{j} x_{j} F_{D}\left(a, e_{j}+b, c+1 ; x\right) F_{D}\left(1-a, e_{j}-b, 2-c ; x\right) .
\end{aligned}
$$

\section{A. Elliott's identity as the twisted period relation}

In Appendix, we show that Elliott's identity among hypergeometric series can be obtained from the twisted period relation (21) in Corollary 1 with detailed calculations.

\section{A.1 Integral representations}

Elliott's identity is given in [BNPV] as

$$
\begin{aligned}
& F\left(\frac{1}{2}+\lambda,-\frac{1}{2}-v, 1+\lambda+\mu ; r\right) F\left(\frac{1}{2}-\lambda, \frac{1}{2}+v, 1+\mu+v ; 1-r\right) \\
+ & F\left(\frac{1}{2}+\lambda, \frac{1}{2}-v, 1+\lambda+\mu ; r\right) F\left(-\frac{1}{2}-\lambda, \frac{1}{2}+v, 1+\mu+v ; 1-r\right) \\
- & F\left(\frac{1}{2}+\lambda, \frac{1}{2}-v, 1+\lambda+\mu ; r\right) F\left(\frac{1}{2}-\lambda, \frac{1}{2}+v, 1+\mu+v ; 1-r\right) \\
= & \frac{\Gamma(1+\lambda+\mu) \Gamma(1+\mu+v)}{\Gamma\left(\lambda+\mu+v+\frac{3}{2}\right) \Gamma\left(\mu+\frac{1}{2}\right)}
\end{aligned}
$$


where $\lambda, \mu, v$ are complex parameters with

$$
1+\lambda+\mu, 1+\mu+v \neq 0,-1,-2, \ldots,
$$

and the main variable $r$ satisfies inequalities $|r|<1$ and $|1-r|<1$. We have

$$
\begin{aligned}
& F\left(\frac{1}{2}+\lambda,-\frac{1}{2}-v, 1+\lambda+\mu ; r\right)=\frac{\Gamma(\lambda+\mu+1)}{\Gamma\left(\lambda+\frac{1}{2}\right) \Gamma\left(\mu+\frac{1}{2}\right)} \int_{0}^{1} t^{\lambda-1 / 2}(1-t)^{\mu-1 / 2}(1-r t)^{v+1 / 2} d t, \\
& F\left(\frac{1}{2}+\lambda, \frac{1}{2}-v, 1+\lambda+\mu ; r\right)=\frac{\Gamma(\lambda+\mu+1)}{\Gamma\left(\lambda+\frac{1}{2}\right) \Gamma\left(\mu+\frac{1}{2}\right)} \int_{0}^{1} t^{\lambda-1 / 2}(1-t)^{\mu-1 / 2}(1-r t)^{\nu-1 / 2} d t, \\
& F\left(\frac{1}{2}-\lambda, \frac{1}{2}+v, 1+\mu+v ; 1-r\right) \\
= & \frac{\Gamma(\mu+v+1)}{\Gamma\left(-\lambda+\frac{1}{2}\right) \Gamma\left(\lambda+\mu+v+\frac{1}{2}\right)} \int_{0}^{1} s^{-\lambda-1 / 2}(1-s)^{\lambda+\mu+v-1 / 2}(1-(1-r) s)^{-v-1 / 2} d s \\
= & \frac{\Gamma(\mu+v+1)}{\Gamma\left(-\lambda+\frac{1}{2}\right) \Gamma\left(\lambda+\mu+v+\frac{1}{2}\right)} \int_{-\infty}^{0}(-t)^{-\lambda-1 / 2}(1-t)^{-\mu-1 / 2}(1-r t)^{-v-1 / 2} d t, \\
& F\left(-\frac{1}{2}-\lambda, \frac{1}{2}+v, 1+\mu+v ; 1-r\right) \\
= & \frac{\Gamma(\mu+v+1)}{\Gamma\left(-\lambda-\frac{1}{2}\right) \Gamma\left(\lambda+\mu+v+\frac{3}{2}\right)} \int_{0}^{1} s^{-\lambda-3 / 2}(1-s)^{\lambda+\mu+v+1 / 2}(1-(1-r) s)^{-v-1 / 2} d s \\
= & \frac{\Gamma(\mu+v+1)}{\Gamma\left(-\lambda-\frac{1}{2}\right) \Gamma\left(\lambda+\mu+v+\frac{3}{2}\right)} \int_{-\infty}^{0}(-t)^{-\lambda-3 / 2}(1-t)^{-\mu-1 / 2}(1-r t)^{-v-1 / 2} d t,
\end{aligned}
$$

under the condition

$$
\lambda, \mu, v, \lambda+\mu+v \notin\left\{\frac{1}{2}+n \mid n \in \mathbb{Z}\right\},
$$

where we use the regularization of $(0,1)$ or $(-\infty, 0)$ with respect to the integrand if necessary. Here note that a variable change $s=t /(t-1)$ is used.

\section{A.2 Setting of a local system}

Hereafter we assume the conditions (23) and (24), and we set

$$
\begin{gathered}
u(t)=t^{1 / 2+\lambda}(1-t)^{-1 / 2+\mu}(1-r t)^{1 / 2+v}, \\
\varphi_{1}=\frac{d t}{t}, \quad \varphi_{2}=\frac{d t}{t(1-r t)}=\left(\frac{1}{t}-\frac{1}{t-1 / r}\right) d t, \\
\psi_{1}=\frac{d t}{1-t}=\frac{-d t}{t-1}, \quad \psi_{2}=\frac{d t}{t(1-t)}=\left(\frac{1}{t}-\frac{1}{t-1}\right) d t .
\end{gathered}
$$

Then we have

$$
1 / u(t)=t^{-1 / 2-\lambda}(1-t)^{1 / 2-\mu}(1-r t)^{-1 / 2-v},
$$

and

$$
\begin{aligned}
& F\left(\frac{1}{2}+\lambda,-\frac{1}{2}-v, 1+\lambda+\mu ; r\right)=\frac{\Gamma(\lambda+\mu+1)}{\Gamma\left(\lambda+\frac{1}{2}\right) \Gamma\left(\mu+\frac{1}{2}\right)} \int_{0}^{1} u(t) \varphi_{1} \\
& F\left(\frac{1}{2}+\lambda, \frac{1}{2}-v, 1+\lambda+\mu ; r\right)=\frac{\Gamma(\lambda+\mu+1)}{\Gamma\left(\lambda+\frac{1}{2}\right) \Gamma\left(\mu+\frac{1}{2}\right)} \int_{0}^{1} u(t) \varphi_{2}
\end{aligned}
$$




$$
\begin{aligned}
F\left(\frac{1}{2}-\lambda, \frac{1}{2}+v, 1+\mu+v ; 1-r\right) & =\frac{\Gamma(\mu+v+1) e^{\pi \sqrt{-1}(\lambda+1 / 2)}}{\Gamma\left(-\lambda+\frac{1}{2}\right) \Gamma\left(\lambda+\mu+v+\frac{1}{2}\right)} \int_{-\infty}^{0} u(t)^{-1} \psi_{1}, \\
& =\frac{\sqrt{-1} \Gamma(\mu+v+1) e^{\pi \sqrt{-1} \lambda}}{\Gamma\left(-\lambda+\frac{1}{2}\right) \Gamma\left(\lambda+\mu+v+\frac{3}{2}\right)}\left(\lambda+\mu+v+\frac{1}{2}\right) \int_{-\infty}^{0} u(t)^{-1} \psi_{1}, \\
F\left(-\frac{1}{2}-\lambda, \frac{1}{2}+v, 1+\mu+v ; 1-r\right) & =\frac{\Gamma(\mu+v+1) e^{\pi \sqrt{-1}(\lambda+3 / 2)}}{\Gamma\left(-\lambda-\frac{1}{2}\right) \Gamma\left(\lambda+\mu+v+\frac{3}{2}\right)} \int_{-\infty}^{0} u(t)^{-1} \psi_{2} \\
& =\frac{-\sqrt{-1} \Gamma(\mu+v+1) e^{\pi \sqrt{-1} \lambda}}{\Gamma\left(-\lambda+\frac{1}{2}\right) \Gamma\left(\lambda+\mu+v+\frac{3}{2}\right)}\left(-\lambda-\frac{1}{2}\right) \int_{-\infty}^{0} u(t)^{-1} \psi_{2} .
\end{aligned}
$$

Thus integrals are expressed as

$$
\begin{aligned}
& \left(\int_{0}^{1} u(t) \varphi_{1}, \int_{0}^{1} u(t) \varphi_{2}\right) \\
= & \frac{\Gamma\left(\lambda+\frac{1}{2}\right) \Gamma\left(\mu+\frac{1}{2}\right)}{\Gamma(\lambda+\mu+1)}\left(F\left(\frac{1}{2}+\lambda,-\frac{1}{2}-v, 1+\lambda+\mu ; r\right), F\left(\frac{1}{2}+\lambda, \frac{1}{2}-v, 1+\lambda+\mu ; r\right)\right) ; \\
& \left(\begin{array}{c}
\int_{-\infty}^{0} u(t)^{-1} \psi_{1} \\
\int_{-\infty}^{0} u(t)^{-1} \psi_{2}
\end{array}\right) \\
= & \frac{\Gamma\left(-\lambda+\frac{1}{2}\right) \Gamma\left(\lambda+\mu+v+\frac{3}{2}\right)}{\sqrt{-1} e^{\pi \sqrt{-1} \lambda} \Gamma(\mu+v+1)}\left(\begin{array}{cc}
\frac{1}{\lambda+\mu+v+1 / 2} & 0 \\
0 & \frac{1}{\lambda+1 / 2}
\end{array}\right)\left(\begin{array}{c}
F\left(\frac{1}{2}-\lambda, \frac{1}{2}+v, 1+\mu+v ; 1-r\right) \\
F\left(-\frac{1}{2}-\lambda, \frac{1}{2}+v, 1+\mu+v ; 1-r\right)
\end{array}\right) .
\end{aligned}
$$

\section{A.3 Transform of a twisted period relation into Elliott's identity}

The intersection matrix $H_{c}$ of $\varphi_{1}, \varphi_{2}$ and $\psi_{1}, \psi_{2}$ is

$$
H_{c}=2 \pi \sqrt{-1}\left(\begin{array}{cc}
\frac{1}{1 / 2+\lambda+\mu+v} & \frac{1}{1 / 2+\lambda} \\
0 & \frac{1}{1 / 2+\lambda}
\end{array}\right)
$$

by Theorem 5. We take a basis of the twisted homology group for $u(t)$ and that for $1 / u(t)$ by extending $\gamma$ and $\delta$, respectively, where $\gamma$ and $\delta$ are the twisted cycles given by the regularization of $(0,1)$ with respect to $u(t)$ and that of $(-\infty, 0)$ with respect to $u^{-1}(t)$. The intersection number of these twisted cycles is $\frac{1}{-e^{2 \pi \sqrt{-1}} \lambda}-1$ by Theorem 3 . Consider the $(1,1)$-entry of

$$
{ }^{t} \Pi_{\omega}{ }^{t} H_{c}^{-1} \Pi_{-\omega}=H_{h}
$$

which is the transpose of the second identity of (21) in Corollary 1. Then it yields

$$
\left(\int_{0}^{1} u(t) \varphi_{1}, \int_{0}^{1} u(t) \varphi_{2}\right){ }^{t} H_{c}^{-1}\left(\begin{array}{c}
\int_{-\infty}^{0} \frac{1}{u(t)} \psi_{1} \\
\int_{-\infty}^{0} \frac{1}{u(t)} \psi_{2}
\end{array}\right)=\frac{-1}{e^{2 \pi \sqrt{-1} \lambda}+1}
$$


Rewrite the integrals in the equality (27) in terms of hypergeometric series by (25) and (26). Then its exp and Gamma factors reduce to

$$
\begin{aligned}
& \frac{\Gamma\left(\lambda+\frac{1}{2}\right) \Gamma\left(\mu+\frac{1}{2}\right)}{\Gamma(\lambda+\mu+1)} \cdot \frac{\Gamma\left(-\lambda+\frac{1}{2}\right) \Gamma\left(\lambda+\mu+v+\frac{3}{2}\right)}{\sqrt{-1} e^{\pi \sqrt{-1} \lambda} \Gamma(\mu+v+1)} \\
= & \frac{\Gamma\left(\lambda+\frac{1}{2}\right) \Gamma\left(1-\left(\lambda+\frac{1}{2}\right)\right)}{\sqrt{-1} e^{\pi \sqrt{-1} \lambda}} \cdot \frac{\Gamma\left(\lambda+\mu+v+\frac{3}{2}\right) \Gamma\left(\mu+\frac{1}{2}\right)}{\Gamma(\lambda+\mu+1) \Gamma(\mu+v+1)} \\
= & \frac{\pi}{\sin \left(\pi\left(\lambda+\frac{1}{2}\right)\right)} \cdot \frac{1}{\sqrt{-1} e^{\pi \sqrt{-1} \lambda}} \cdot \frac{\Gamma\left(\lambda+\mu+v+\frac{3}{2}\right) \Gamma\left(\mu+\frac{1}{2}\right)}{\Gamma(\lambda+\mu+1) \Gamma(\mu+v+1)} \\
= & \frac{-2 \pi \sqrt{-1}}{e^{2 \pi \sqrt{-1} \lambda}+1} \cdot \frac{\Gamma\left(\lambda+\mu+v+\frac{3}{2}\right) \Gamma\left(\mu+\frac{1}{2}\right)}{\Gamma(\lambda+\mu+1) \Gamma(\mu+v+1)},
\end{aligned}
$$

and the product of ${ }^{t} H_{c}^{-1}$ and the $2 \times 2$-matrix in (26) reduces to

$$
\frac{1}{2 \pi \sqrt{-1}}\left(\begin{array}{cc}
\frac{1}{2}+\lambda+\mu+\nu & 0 \\
-\left(\frac{1}{2}+\lambda+\mu+\nu\right) & \frac{1}{2}+\lambda
\end{array}\right)\left(\begin{array}{cc}
\frac{1}{1 / 2+\lambda+\mu+\nu} & 0 \\
0 & \frac{1}{1 / 2+\lambda}
\end{array}\right)=\frac{1}{2 \pi \sqrt{-1}}\left(\begin{array}{cc}
1 & 0 \\
-1 & 1
\end{array}\right) .
$$

Hence the equality (27) is transformed into

$$
\begin{aligned}
& \left(F\left(\frac{1}{2}+\lambda,-\frac{1}{2}-v, 1+\lambda+\mu ; r\right), F\left(\frac{1}{2}+\lambda, \frac{1}{2}-v, 1+\lambda+\mu ; r\right)\right) \\
& \cdot\left(\begin{array}{cr}
1 & 0 \\
-1 & 1
\end{array}\right) \cdot\left(\begin{array}{c}
F\left(\frac{1}{2}-\lambda, \frac{1}{2}+v, 1+\mu+v ; 1-r\right) \\
F\left(-\frac{1}{2}-\lambda, \frac{1}{2}+v, 1+\mu+v ; 1-r\right)
\end{array}\right) \\
= & \frac{\Gamma(\lambda+\mu+1) \Gamma(\mu+v+1)}{\Gamma\left(\lambda+\mu+v+\frac{3}{2}\right) \Gamma\left(\mu+\frac{1}{2}\right)},
\end{aligned}
$$

which is equivalent to Elliott's identity (22).

\section{Acknowledgments}

The author could not be present the work shop

"MathemAmplitudes 2019: Intersection Theory \& Feynman Integrals"

This article is based on author's talks in seminars in the University of Padova during the period September 16-19, 2019, held as a kick-off meeting, anticipating the workshop. The author expresses his gratitude to the organizers for inviting me to contribute to these proceedings, and to the speakers of the work shop for acknowledging my contribution.

Professor Pierpaolo Mastrolia had conjectured the main result in Appendix, that is, Elliott's identity can be obtained from the twisted period relation, and explained his conjecture together with its importance in theoretical physics to the author in the kick-off meeting. The author is grateful to him for his explanation, and glad to have several fruitful discussions with the members of his research team in this meeting. 


\section{References}

[AK] K. Aomoto and M. Kita, Theory of hypergeometric functions, With an appendix by Toshitake Kohno, Translated from the Japanese by Kenji Iohara, Springer Monographs in Mathematics. Springer-Verlag, Tokyo, 2011.

[BNPV] R. Balasubramanian, S. Naik, S. Ponnusamy and M. Vuorinen, Elliott's identity and hypergeometric functions, J. Math. Anal. Appl., 271 (2002), 232-256.

[CM] K. Cho and K. Matsumoto, Intersection theory for twisted cohomologies and twisted Riemann's period relation I, Nagoya Math. J., 139 (1995), 67-86.

[KM] M. Kita and K. Matsumoto, Duality for Hypergeometric Functions and Invariant GaussManin Systems, Compositio Math., 108 (1997), 77-106.

[KN] M. Kita and M. Noumi, On the structure of cohomology groups attached to integrals of certain many valued analytic functions, Japan. J. Math., 9 (1983), 113-157.

[KY1] M. Kita and M. Yoshida, Intersection theory for twisted cycles, Math. Nachr., 166 (1994), 287-304

[KY2] M. Kita and M. Yoshida, Intersection theory for twisted cycles II, Math. Nachr. Math. Nachr., 168 (1994), 171-190.

[MMT] H. Majima, K. Matsumoto and N. Takayama, Intersection theory for confluent hypergeometric functions, Tohoku J. Math., 52, (2000), 489-513.

[M1] K. Matsumoto, Intersection numbers for logarithmic k-forms, Osaka J. Math., 35 (1998), 873-893.

[M2] K. Matsumoto, Intersection numbers for 1-forms associated with confluent hypergeometric functions, Funkcial. Ekvac., 41 (1998), 291-308.

[M3] K. Matsumoto, Relative twisted homology and cohomology groups associated with Lauricella's $F_{D}$, Algebraic Geometry (math.AG), arXiv:1804.00366v2, 2019.

[MY] K. Matsumoto and M. Yoshida, Recent progress of intersection theory for twisted (co)homology groups, Advanced studies in Pure Mathematics 27, 2000, Arrangements - Tokyo 1998, 217-237.

[Y1] M. Yoshida, Fuchsian differential equations, Aspects of Mathematics, E11, Vieweg \& Sohn, Braunschweig, 1987.

[Y2] M. Yoshida, Hypergeometric functions, my love, Aspects of Mathematics, E32, Vieweg \& Sohn, Braunschweig, 1997. 